\title{
Challenges and procedures for experiments with steady and unsteady model velocities in a water towing tank
}

\author{
M. Jentzsch ${ }^{1}$ (1) H. -J. Schmidt ${ }^{1} \cdot$ R. Woszidlo ${ }^{1} \cdot$ C. N. Nayeri ${ }^{1} \cdot$ C. O. Paschereit ${ }^{1}$
}

Received: 12 June 2020 / Revised: 17 November 2020 / Accepted: 18 January 2021

(c) The Author(s) 2021

\begin{abstract}
Challenges for steady and unsteady model motion in a large water towing tank and procedures to overcome them are the focus of the presented work. Some challenges are attributed to experiments conducted in water, whereas others are uniquely ascribed to a towing tank facility. Data convergence and outlier detection are studied based on the phase averaged pressure in order to ensure proper data quality. Pressure measurements are performed with non-surface mounted sensors. Therefore, inertia effects are detrimental when the attached tubing is not fully de-aired. A procedure for de-airing the pressure sensor cavity and its tubing is described. An iterative approach is developed that compensates for nonlinear distortion of the model's velocity profile. Further, vibration effects are examined by distinguishing mechanical and flow-induced frequencies that scale with the instantaneous model velocity. Sloshing waves are excited, which are a function of the water basin size. The first sloshing mode defines the required sensor offset time in between test cases when prevailing sloshing waves have not fully decayed. This appropriate selection of sensor offset time reduces data scatter and enables a reasonable waiting time in between test cases. A skim plate installed just below the water surface offers a potential solution to alleviate surface wave effects over the model.
\end{abstract}

\section{Introduction}

Towing tanks are often used to investigate the hydrodynamics of boats, ships, and underwater vehicles. However, a towing tank facility is also an ideal choice to study the aerodynamics of bodies such as wings, blades, or bluff bodies. These geometries operate not only in steady, but also unsteady, environments.

There are many advantages of using a water towing tank rather than a wind tunnel. The model is towed through quiescent water, which is advantageous to test with realistic boundary conditions. Compared to air, the kinematic viscosity of water is smaller by a factor of about 15 , yielding higher REYNolds numbers at lower velocities. Simultaneously, the time scales of flow structures are increased, thus leading to improved determination of transient effects with more ease and accuracy (Greenblatt et al. 2016; Schmidt et al. 2017). The density of water is about 800 times larger,

M. Jentzsch

marvin.jentzsch@tu-berlin.de

1 Institute of Fluid Dynamics and Technical Acoustics, Hermann-Föttinger-Institut, Technische Universität Berlin, Berlin 10623, Germany hence amplifying pressure, forces, and moments acting on the model. The generation of arbitrary velocity profiles is easier to produce in a towing tank than in a wind tunnel. This is achieved by controlling electric motors and moving the model, whereas in wind tunnels, it is necessary to move the bulk of fluid. Thus, the surge motion is analyzed with more ease. Compressibility effects are absent, which simplifies the problem. However, applications that need to account for compressibility effects cannot be studied in a water towing tank.

Despite obvious advantages, many challenges exist with tests conducted in a water towing tank. Several guidelines and recommendations regarding uncertainty analysis, instrument calibration, particle image velocimetry (PIV) measurements, and best practices are provided in the proceedings of the International Towing Tank Conference (ITTC) (International Towing Tank Conference 2020) and by Gad-el-Hak (1987). The present paper extends these guidelines for the consideration of submerged airfoil models at steady and unsteady velocities tested in a large water towing tank, and documents the related challenges that have not been subject of other publications. Pertinent literature, if available, is provided within each individual section. 
The topic of data quality is assessed in Sect. 3.1 by means of phase averaged quantities to determine data convergence and to detect outliers. Section 3.2 addresses challenges associated with non-surface mounted pressure sensors and provides procedures to enable their proper use. Section 3.3 provides an iterative approach to obtain accurate velocity profiles of arbitrary shape. Furthermore, a vibration analysis is carried out to distinguish mechanical from flow-induced frequencies. Last, an overview of challenges and suggested solutions related to free surface effects is provided in Sect. 3.4.

A priori knowledge of the discussed topics can help to properly design the test rig and model. The suitability for aerodynamic investigations is illustrated, especially for experiments with unsteady freestream velocity since these are rarely addressed in the literature. Procedures and suggestions are provided to overcome various challenges. Many findings are also transferable to experiments in a water tunnel. Researchers are encouraged to assess their facility for these phenomena and document their means to address them.

\section{Setup and instrumentation}

The towing tank facility in which the experiments were carried out is described in Sect. 2.1. It was built in 1903 and is one of the largest towing tanks in Europe. Section 2.2 summarizes details about the flat plate model and the test rig used to install two-dimensional models.

\subsection{Towing tank facility}

The large water towing tank at the Technische Universität (TU) Berlin has a water basin length of $250 \mathrm{~m}$, a width of $8.1 \mathrm{~m}$, and an average depth of $4.8 \mathrm{~m}$. The basin depth drops from 3.4 to $5.2 \mathrm{~m}$ at a longitudinal position of $60 \mathrm{~m}$. A preparation area with a length of $17 \mathrm{~m}$, width of $2.1 \mathrm{~m}$, and depth of $2.4 \mathrm{~m}$ can be decoupled from the main water basin using a bulkhead and drained via an electrical pump. The preparation area is used to install the test rig and model. A carriage train with a weight of about 25 tons moves on two rails that are mounted along the walls of the water basin. The rails are carefully leveled with the water surface to maintain a constant distance between measurement platform and water. The test rig and model are mounted to a measurement platform that can be traversed in vertical and lateral direction. Loads of two tons in vertical (lift) and one ton in longitudinal (drag) direction can be applied. Application of side loads is avoided to prevent the carriage train from derailing. A sketch of the towing tank facility and carriage train is shown in Fig. 1. Eight $55.5 \mathrm{~kW}$ DC-motors are installed to move the carriage train with computer controlled velocity profiles of arbitrary shape. The maximum velocity that can be obtained is $12.5 \mathrm{~m} \mathrm{~s}^{-1}$ with a maximum acceleration of $1 \mathrm{~m} \mathrm{~s}^{-2}$. The carriage velocity is measured using a rotary encoder with

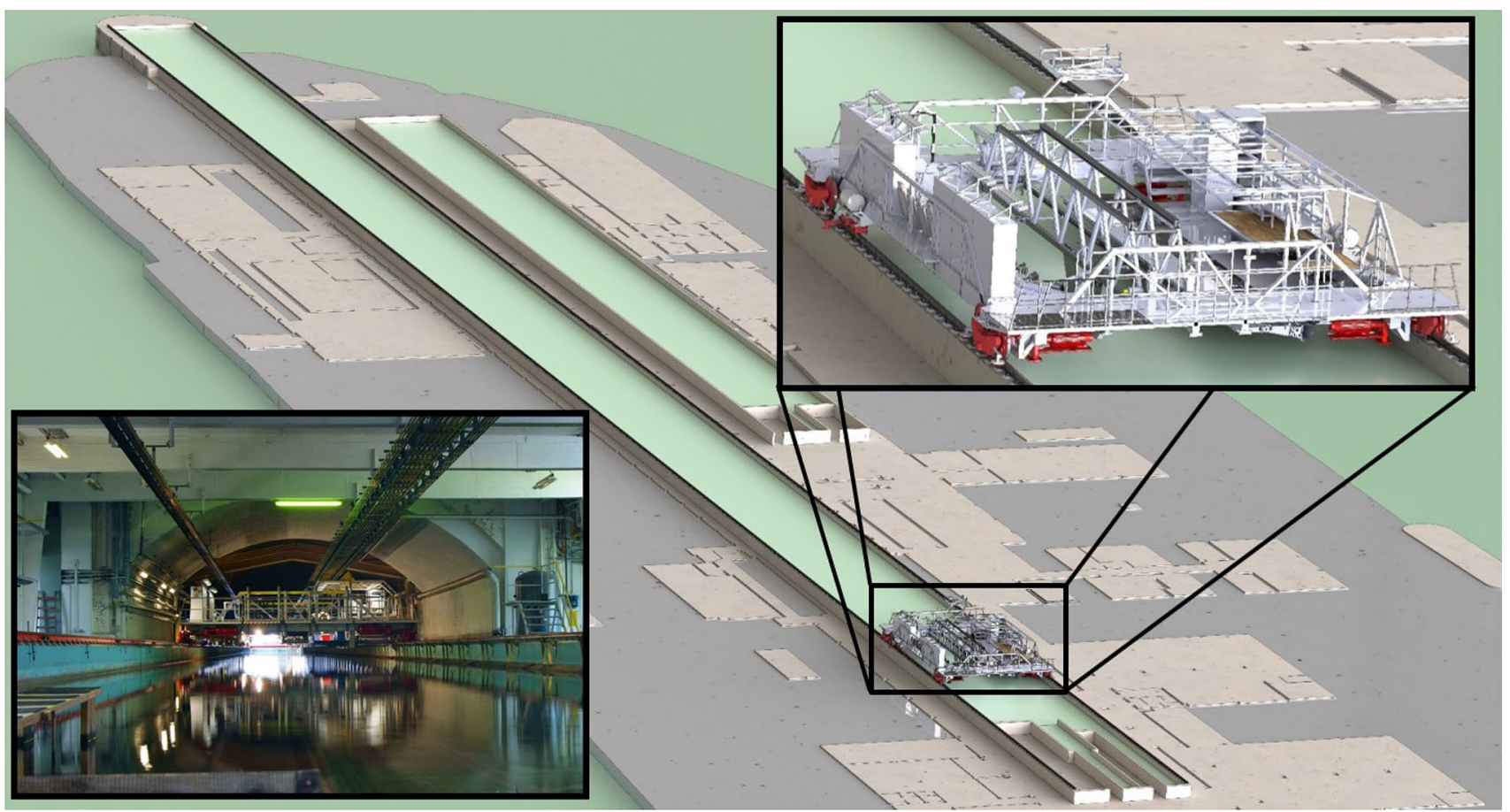

Fig. 1 Towing tank and towing carriage at the TU Berlin 
a resolution of 10,000 ticks per meter in combination with a frequency-to-current signal converter (WAS4 PRO Freq) by Weidmueller.

\subsection{Test rig setup and flat plate model}

A test rig is used to install two-dimensional models with a span of $s=1.0 \mathrm{~m}$ to the measurement platform (Fig. 2). Splitter plates on both sides of the model provide a quasitwo-dimensional flow field over the model. The splitter plate dimensions are $1.25 \mathrm{~m} \times 1.0 \mathrm{~m} \times 0.035 \mathrm{~m}(1 \times \mathrm{w} \times \mathrm{t})$. Four steel frameworks (two fore and two aft) are used to attach the splitter plates. The frameworks consist of multiple welded plates with a thickness of 5 to $8 \mathrm{~mm}$ oriented at an angle to each other but at zero incidence with respect to the longitudinal carriage motion. Angular steel mounts with a welded pin of $50 \mathrm{~mm}$ diameter are used on both sides to mount the flat plate model. The shafts are inserted into bearings located on the outer part of either splitter plate such that the angle of incidence can be changed continuously. The angle of attack is held firmly by end plates on the outer side of the test rig. The submersion depth of the model $d_{\text {model }}$ is controlled by traversing the measurement platform, and thus the entire test rig in vertical direction. The submergence of the model ranges from $1.3 c$ to $2.5 c$ below the water surface measured from the upper side of the model at an angle of attack of $\alpha=0^{\circ}$, where $c$ is the chord length of the model.
A flat plate model serves as a generic surrogate for the current effort to investigate the experimental challenges associated with towing tank experiments for steady and unsteady airfoil aerodynamics. The model has an elliptical leading edge and a blunt trailing edge. The span measures $s$ $=0.95 \mathrm{~m}$ and the chord $c=0.5 \mathrm{~m}$ yielding an aspect ratio of $A R=1.9$. The two-dimensionality of the flow is analyzed by two spanwise pressure sensors located at $y /(s / 2)=0.25$ and $y /(s / 2)=0.5$ and $x / c=0.55$. These recordings are compared to the pressure sensor reading obtained from the pressure tap located at the same $x / c$ position at mid-span $(y /(s / 2)=0)$. The spanwise deviation depends on various parameters such as angle of attack, REYNOLDs number, whether a skim plate is installed, and velocity profile. The deviation is calculated for an angle of attack of $\alpha=10^{\circ}$, which is near stall, submergence depth of $d_{\text {model }}=2.5 \mathrm{c}$, and skim plate installed at $d_{\mathrm{SP}}=0.2 c$. For steady velocity tests at $R e_{0} \approx 1,320,000$, the mean pressure coefficient deviation $\left(\Delta \overline{C_{p}}=\overline{C_{p, y /(s / 2)=0}}-\overline{C_{p, y /(s / 2) \neq 0}}\right)$ yields $\Delta \overline{C_{p, y /(s / 2)=0.25}}=0.02$ and $\Delta C_{p, y /(s / 2)=0.5}=0.11$ with the time-averaged mid-span value being $\overline{C_{p, y /(s / 2)=0}}=-0.40$. For unsteady tests at $R e_{0} \approx 440,000, k \approx 0.24$, and $\sigma=0.5$, the root-mean-squared deviation (RMSD) of the phase averaged pressure coefficient is $\operatorname{RMSD}\left(C_{p, y /(s / 2)=0.25}\right)=0.05$ and $\operatorname{RMSD}\left(C_{p, y /(s / 2)=0.5}\right)=0.09$. Deviations for the unsteady measurements remain acceptable because unsteady effects dominate the flow field. This confirms the findings for unsteady measurements in a wind tunnel of low aspect ratio

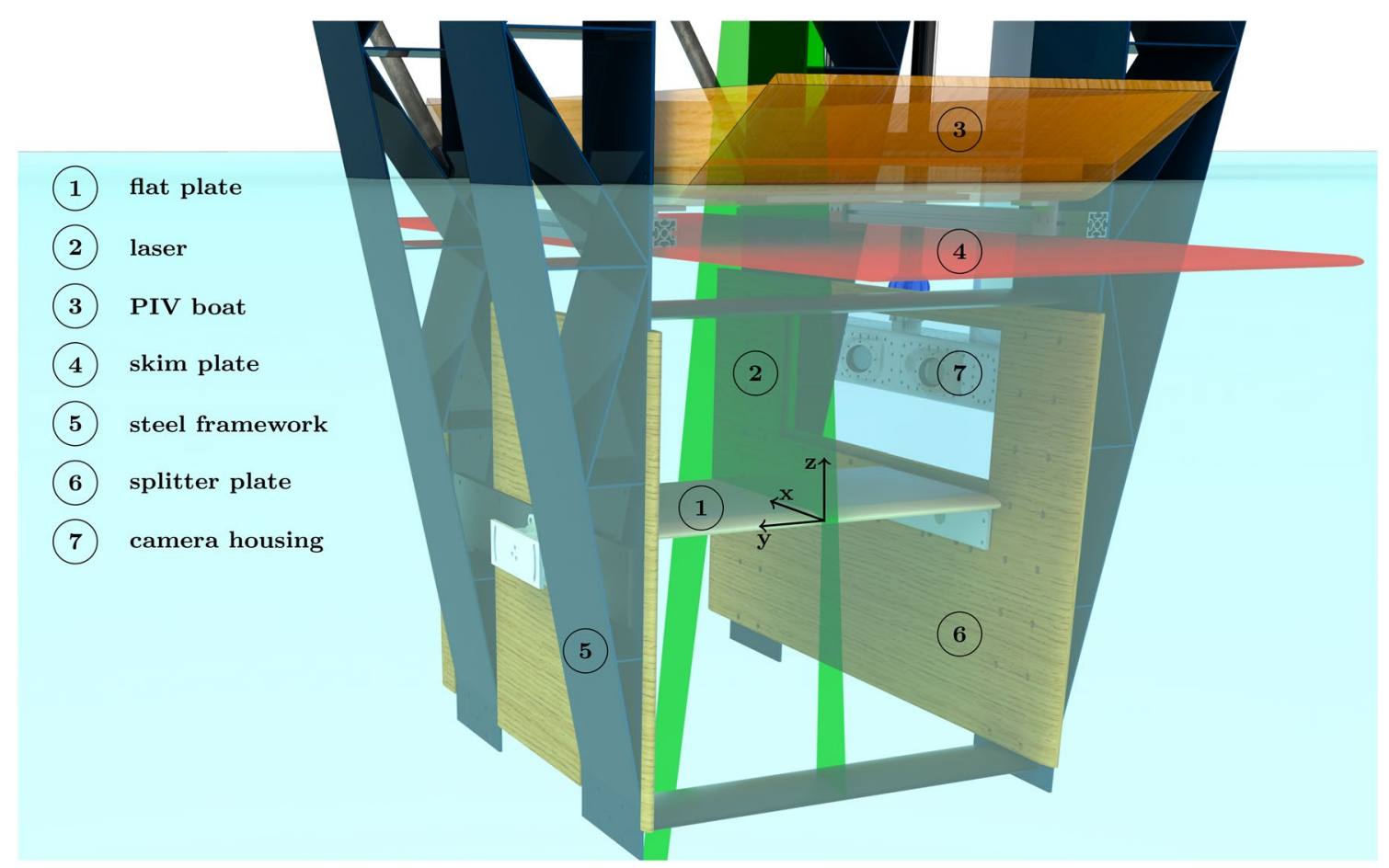

Fig. 2 Test rig for two-dimensional models with PIV system, skim plate, and model installed 
airfoils made by Carr et al. (1977) and McAlister et al. (1978). The thickness of the model is $t=0.03 \mathrm{~m}$, and the length of the semi-major axis of the elliptical leading edge is $a=0.06 \mathrm{~m}$. The model is a composite of coated wood and aluminum. Two aluminum plates $(0.01 \mathrm{~m}$ and $0.005 \mathrm{~m}$ thick) are mounted on top and bottom of the wood core in order to increase the model's structural integrity.

The use of plain aluminum parts submerged in water is not an optimal choice due to pitting corrosion that forms salt-like crystals and tiny holes. Those crystals accumulate and grow over time, potentially tripping the flow over the model's surface. The corrosion occurs for a specific alloy/ environment combination, and therefore, it is advised to carry out a material study with small samples before building sophisticated models. Alternatively, the aluminum can be powder coated to add a resistive surface layer. The wooden core of the model is coated with two-component epoxy-resin and thereafter painted with spar varnish to make it water resistant. In order to place the pressure sensors, tubing, and wiring into the model, pockets and channels are milled into the wood core.

The pressure sensor tubing connecting pressure port and sensor is kept as short as possible $(0.15 \mathrm{~m})$ and equal for all sensors to avoid dynamic pressure alteration in amplitude and phase. Based on the equations provided by Bergh and Tijdeman (1965) with a conservative estimate for the transducer volume of the pressure sensor, the resonance frequency of this sensor-tube system is around $f \approx 400 \mathrm{~Hz}$, whereas the expected flow-induced shedding frequencies of the model in water are at least one order of magnitude lower. A total of 15 pressure taps are incorporated into the flat plate model. Around mid-span seven pressure ports are placed in a staggered fashion into the leading edge (six on top and one on the bottom surface), and six taps are placed sparsely over the remaining $88 \%$ of the model's chord. Two more taps are distributed in spanwise direction as mentioned earlier.

For the current study, differential pressure sensors made by Honeywell (26PCBFA6D) with a range of $\pm 35 \mathrm{kPa}$ and an accuracy of $0.25 \%$ full scale are used together with custom-made amplifiers. The pressure reference side is connected to atmospheric pressure outside of the water. A pressure calibrator can be connected to the tubing of the reference side. The pressure side of the differential pressure sensor measures the surface pressure of the model underwater, and hence, the tubing has to be carefully filled with water. The model's angle of attack is measured using a digital water level with an accuracy of $\pm 0.1^{\circ}$ to in situ calibrate an accelerometer installed within the model. Additionally, a skim plate can be installed above the model (see Fig. 2). The skim plate is $2.44 \mathrm{~m}$ long, $0.96 \mathrm{~m}$ wide, and $0.02 \mathrm{~m}$ thick. The position can be traversed in the longitudinal and vertical directions. For the current study, it is centered above the flat plate model and submerged $0.1 \mathrm{~m}$ below the water surface.
Even though no PIV results are discussed in this document, the PIV setup as used in a current benchmark test is depicted in Fig. 2. It serves as a reference for a potential PIV setup in a towing tank where the PIV system is moving with the model. A more detailed description of the PIV setup is provided by Schmidt et al. (2017). Some examples for other PIV setups in a water towing tank include (Tukker et al. 2007; Gui et al. 2001; Chen and Chang 2006; Jacobi et al. 2016; Corkery et al. 2018; Wilhelmi et al. 2018; Cleaver et al. 2013). Recommended procedures and guidelines for PIV measurements carried out in water are provided by the ITTC (ITTC 7.5-01-03-03 2008; ITTC 7.5-01-03-04 2014).

An ultrasound sensor (General Acoustics, UltraLab USS2001300) is used to record surface wave deflection during and in between test cases. Three triple-axis accelerometers (ADXL335) with a range of $\pm 3 g$ can be attached everywhere on the carriage train in order to capture rig vibration. A fast-acting adhesive is used to ensure a rigid connection between the accelerometer and the surface it is attached to. All time-resolved signals such as pressure, model velocity, acceleration, surface deflection, and PIV camera trigger signal are acquired with a data acquisition system with 32 synchronized channels at a sampling rate of $f_{s}=10,000 \mathrm{~Hz}$. The system consists of a NI 9188 mainframe by National Instruments equipped with eight analog input modules (NI 9215 (BNC)).

\section{Results}

Before exploring the challenges related to steady and unsteady measurements in a towing tank, general considerations such as governing equations and cavitation are summarized. The unsteadiness of the flow field is quantified by the reduced frequency $k$ (Eq. 1), where $\omega$ is the angular frequency, $c$ the chord length of the model, and $U_{0}$ the average velocity of the freestream or model.

$k=\frac{\omega c}{2 U_{0}}=\frac{\pi f c}{U_{0}}$

The flow is considered steady when $k=0$ or quasi-steady for $k<O(0.01)$ and unsteady for $k>O(0.01)$ (Leishman 2002). When using a towing tank to produce oscillatory surging flows, the achievable range of reduced frequencies is limited by the carriage acceleration. The desired velocity and acceleration profile for the oscillatory surge motion in the longitudinal direction is expressed in Eqs. 2 and 3, where $\sigma$ is the velocity amplitude of the sinusoidal profile.

$U(t)=U_{0}(1+\sigma \sin (\omega t))$ 
$a(t)=U_{0} \sigma \omega \cos (\omega t)=U_{0} \sigma 2 \pi f \cos (\omega t)$

The maximum feasible forcing frequency (Eq. 4) can now be determined by considering the maximum carriage train acceleration (Eq. 3).

$f_{\max }=\frac{a_{\max }}{2 \pi U_{0} \sigma}$

Based on the definition of the reduced frequency (Eq. 1), an expression for maximum achievable reduced frequency is derived as a function of model dimension $c$, maximum acceleration $a_{\max }$, mean velocity $U_{0}$, and relative velocity amplitude $\sigma$ (Eq. 5).

$k_{\max }=\frac{\pi f_{\max } c}{U_{0}}=\frac{\pi c a_{\max }}{2 \pi U_{0}^{2} \sigma}=\frac{c a_{\max }}{2 U_{0}^{2} \sigma}$

Another important effect that needs to be considered when conducting experiments in water is the occurrence of cavitation. Local pressure changes on surfaces are influenced by the model's geometry and local fluid velocity. As a first estimate, a critical pressure coefficient $\left(C_{p, \text { crit }}\right)$ value at which cavitation occurs can be calculated using Eq. 6:

$C_{p, \text { crit }}=\frac{p_{\text {vapor }}-\left(p_{\text {atm }}+p_{\text {hydro }}\right)}{0.5 \rho U_{0}^{2}}$

The flow velocity at which cavitation is expected can be calculated from a known vapor pressure $p_{\text {vapor }}$, atmospheric pressure $p_{\text {atm }}$, and hydrostatic pressure $p_{\text {hydro }}$ at the model's submersion depth $d_{\text {model }}$. Hydrophone and optical measurements can be used for cavitation detection. As a result of cavitation considerations, and also due to the limited length of a towing tank, it is desirable to keep the model's velocity at a minimum and to increase the model's size to increase the REYNOLDS numbers. Furthermore, the model's submersion depth can be maximized to delay the occurrence of cavitation to higher local velocities due to higher hydrostatic pressures.

The following sections address the various challenges that are associated with towing tank experiments for steady and unsteady measurements. The data quality is assessed in Sect. 3.1. Section 3.2 elucidates challenges associated with unsteady pressure measurements using non-surface mounted sensors. Carriage train and setup-related challenges are discussed in Sect. 3.3. The effects of surface waves on measurement procedures and data quality are considered in Sect. 3.4.

\subsection{Data quality}

For experiments in a towing tank, the basin length is limited and waiting times in between test cases are necessary to reduce the turbulence level and surfaces wave deflection.
This limits the measurement time. When (phase) averaging is applied, data quality is enhanced by increasing the number of data sets because it reduces stochastic noise. Experimental campaigns in a towing tank are more time intensive compared to continuously blowing wind tunnel tests. The balance of total testing time and data quality is crucial to reach a high test efficacy. Finding the optimum point where sufficient data quality is obtained within the least testing time is challenging. Testing time can be reduced by either limiting the number of test cases or reducing settling time. However, both methods have an adverse effect on data quality. The factors influencing these quantities and their interdependency are illustrated in Fig. 3. The flow chart does not cover all possible scenarios, but gives an overview about the challenges. The total testing time represents the overall time necessary to test one configuration, which is comprised of the time for the number of runs and the settling time in between each run. The settling time represents the main contributor to the testing time and therefore requires special consideration. A turbulence intensity of zero is not achievable from a practical point of view. Therefore, it is necessary to define a threshold that ensures acceptable data quality, while fluctuations remain in the water tank. The time to reach this value is dependent on many parameters (i.e., turbulence generated over time during and following each test case). Monitoring the water surface deflection may be a viable means for settling time optimization. More details are provided in Sect. 3.4.1.

Data quality is quantified by the signal-to-noise ratio (SNR). Ensemble averaging is applied on periodic flows to increase the SNR. Stochastic noise decreases with $\sqrt{N}$ where $\mathrm{N}$ is the number of data sets for the averaging process (Wulff 2006). Therefore, an increase in data quality is linked to an increase in testing time. A threshold value is necessary as an indication for sufficient data quality such that measurements are only conducted as long as necessary. It is also directly linked to the desired temporal resolution of the results. For example, the oscillatory surge motion is naturally divided into its periods and phase averaged to capture the coherent parts of the signal. The phase averaged signal ranges from $0^{\circ} \leq \phi<360^{\circ}$. It is further subdivided into smaller bins that depend on the desired temporal resolution $\Delta \phi$. The number of data points per bin is dependent on the bin size and total number of usable data points. The SNR can be enhanced by increasing $\Delta \phi$ at the expense of temporal resolution or by acquiring additional data leading to longer total testing time. Details on the phase averaging method for periodic flows are provided in Sect. 3.1.1.

The usable data are the result of the total number of data points acquired with outliers removed. Outliers are a matter of definition but are regarded as data points that do not fit to the bulk of the observations. They can be 


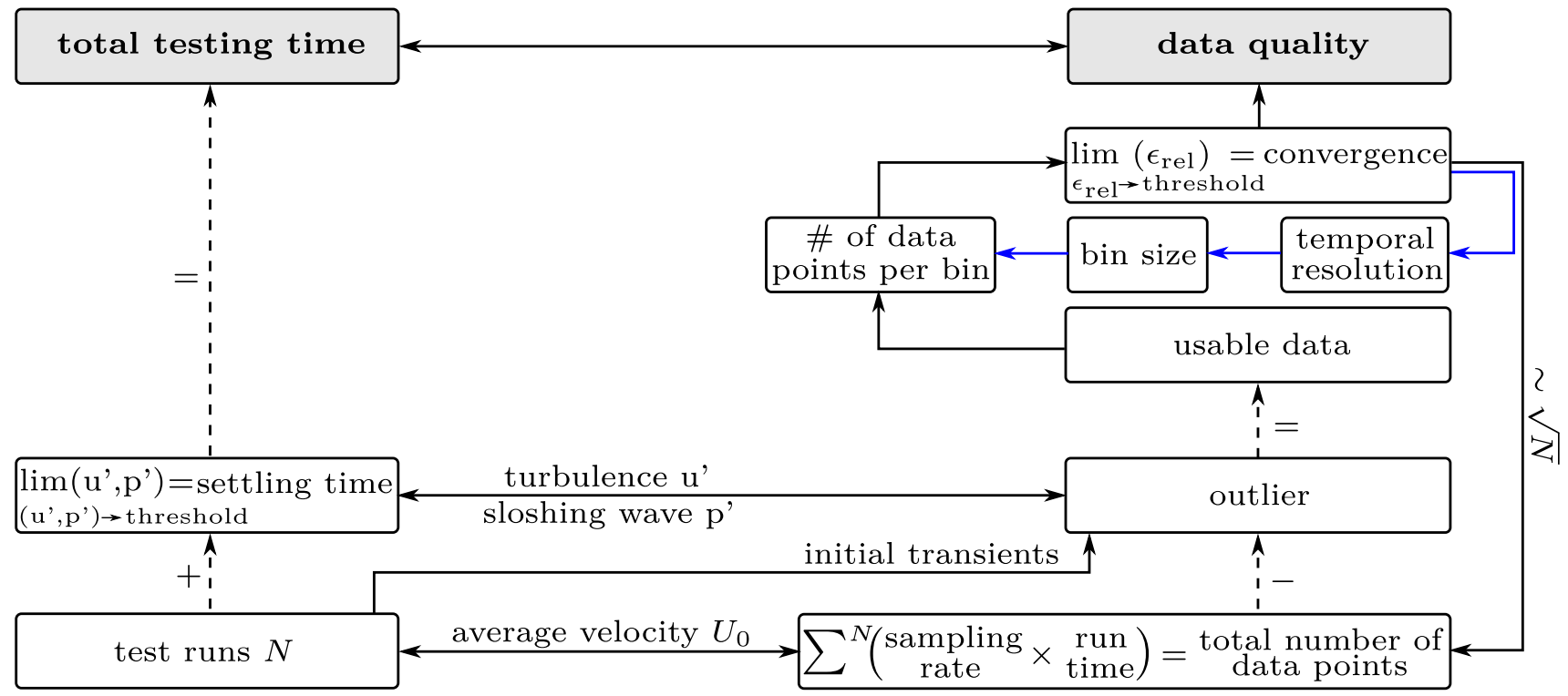

Fig. 3 Flow chart depicting challenges regarding total testing time and data quality for measurements in a towing tank

deterministic (e.g., initial transients) or occur randomly (e.g., perturbations in fluid). A procedure for outlier detection is discussed in Sect. 3.1.2.

The total number of data points for one measurement configuration is the sum of all test runs where the sampling rate multiplied with the run time yields the number of data points per run. The run time is dependent on the average velocity $U_{0}$ and available basin length.

It is challenging to find the optimum in data quality and testing time since they are interdependent. Predefined metrics are required to standardize experiments conducted in a towing tank that assure sufficient and repeatable data quality. The standard procedure for most experiments relies on best practices and the experience of the researcher. For water towing tanks, each facility and each experiment will require its own process to optimize data acquisition time and data quality.

\subsubsection{Phase averaging}

Phase averaging is applied to various signals to capture the coherent parts of the flow behavior. Thus, phase averaging acts as a filter where stochastic noise is removed. The carriage velocity is used as the reference signal to obtain instantaneous velocity and phase information. Thereafter, the signal is divided into its number of periods. The extracted periods are further subdivided into equidistant time and phase steps ranging from $0^{\circ} \leq \phi<360^{\circ}$. This is justified by sampling all channels at a sampling rate of $10,000 \mathrm{~Hz}$, which is at least three orders of magnitude higher than the velocity profile oscillations. The phase average is obtained by calculating the mean at each phase instance across all periods. Deterministic noise is removed a priori to phase averaging. An example of deterministic noise is the start-up effect. When accelerating a model, especially an airfoil from rest, a starting vortex develops at the leading and trailing edge. These vortices affect the circulatory forces by introducing vortex-induced lift. This effect takes time to dissipate (Stevens et al. 2016). A converged state may never be reached depending on the available towing tank length (Mancini et al. 2015). Therefore, it is recommended to always measure data over the entire range of motion to capture initial transient effects. Within the presented study, the first period of a test run often differs in shape and magnitude compared to the following periods, which is dependent on the chosen parameter configuration. Multiple tests are carried out at different parameters (i.e., $\alpha, R e, k, \sigma)$ and show that start-up effects do not carry over into the subsequent periods of oscillation. Therefore, it is not recommended to conduct tests where only one period of oscillation can be obtained.

Phase averaging is not just relevant for measurements with high sampling rates but especially for measurements with low sampling rates such as snapshot PIV. The underwater PIV system to be used in future work is able to capture double frames at a maximum frequency of $6 \mathrm{~Hz}$. This results in a coarse grid of PIV snapshots compared to the time-resolved pressure and velocity data even though time scales in water are increased and forcing frequencies are low. Pointwise averaging is only applicable if the PIV snapshots are acquired phase locked with the reference velocity. 
Phase locked measurements are carried out only if distinct phase instances are of interest. However, these instances of interest are often not known beforehand and information about the flow field over the entire motion period is desired. A high-speed PIV system is most suitable to obtain the time-resolved flow field. However, a high-speed system may not always be available or may not fit within the setup constraints of the towing tank.

Nevertheless, time-resolved flow field information can be extracted from randomly distributed data captured with a low-frequency snapshot PIV system. PIV snapshots that are not phase locked with the carriage velocity are reconstructed in phase using the carriage velocity as the reference signal and the PIV trigger signal. Each snapshot is assigned with a distinct phase value in the range of $0^{\circ} \leq \phi<360^{\circ}$. Snapshots are averaged over predefined bin sizes to increase the SNR. The bin size has to be determined for each individual experiment. For a given amount of snapshots available, there exists an optimal choice of window size as described by Ostermann et al. (2015). This reconstructed and phase averaged PIV field can be treated as time-resolved and compared to other phase averaged quantities such as pressure or force. Methods such as the calculation of the finite-time Lyapunov exponent (FTLE) or dynamic mode decomposition (DMD) are applicable to the quasi-time-resolved PIV velocity field.

\subsubsection{Outlier detection and convergence}

Outliers for the oscillatory surge motion are detected based on the time-resolved pressure signal. The pressure sensor $(x / c=0.01)$ closest to the pressure suction peak is chosen for this analysis because it is most susceptible to changes. The phase averaged signal $x$ of all periods is calculated. Thereafter, the normalized cross-correlation coefficient $r$ with zero lag of the phase averaged quantity with each individual period $y$ is computed according to Eq. 7, where $N$ defines the number of data points in one period. The period with least correlation to the phase average (largest deviation to a value of 1 ) is removed, and the procedure is repeated iteratively. After each iteration, the phase averaged signal is recomputed using the remaining periods. Thereafter, the root mean square error (RMSE) of successive phase averaged signals is calculated. The RMSE value increases when outliers are included in the calculation or when too many periods are removed, thus decreasing the SNR. The minimum of the obtained RMSE curve (Fig. 4, red dot) indicates how many periods are kept for calculating the phase averaged pressure yielding the highest SNR. All discarded periods are referred to as outliers. However, this method requires acquisition of sufficient periods in order to be statistically robust. Appropriate filtering is applied based on a vibration analysis (Sect. 3.3.2) before the outlier detection scheme is applied.

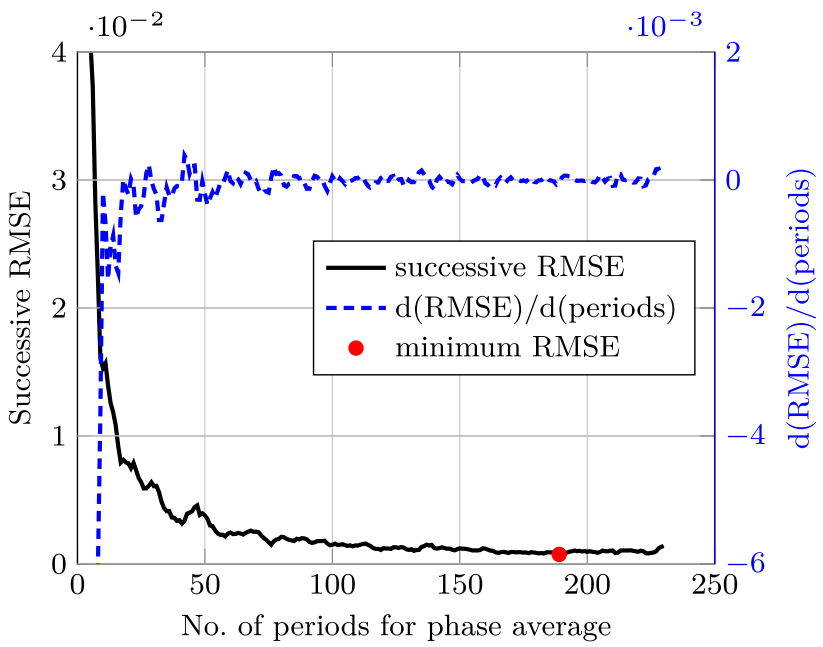

Fig. 4 Convergence and outlier detection based on the phase averaged signal of a pressure sensor at $x / c=0.01$ (ten repeated test runs at $\alpha=10^{\circ}, R e_{0} \approx 440,000, k \approx 0.24, \sigma=0.5$ )

This enhances the detection of outliers since coherent noise (e.g., motor vibration) is removed, thus not influencing the cross-correlation.

$r=\frac{\sum_{n=1}^{N} x(n) y(n)}{\sum_{n=1}^{N} x(n)^{2}}$

As an example for a convergence study, a total of 230 periods is acquired within ten repeated test cases at $\alpha=10^{\circ}$ (Fig. 4). The RMSE value is declining and converging until a minimum is reached (red dot). The rate of change of the RMSE value flattens out quickly, and minor improvements on the SNR are achieved with increasing number of periods. A threshold can be defined to satisfy sufficient data quality of the phase averaged signal. This threshold may be dependent on the individual experiment. For the presented case, two repetitions of a test run with 23 periods each were found to be sufficient. This process does not just ensure sufficient data quality but also avoids unnecessary testing time.

\subsection{Pressure measurements}

Pressure measurements in water can be challenging compared to measurements in air. The sensor cavity and attached tubing have to be completely de-aired. Furthermore, models are often too small to integrate the sensors and tubing, and hence, literature on pressure measurements in water is sparse. Independent of the working fluid, it is desirable to use surface mounted pressure sensors for dynamic pressure measurements. However, it is often not practical to embed sensors into the model or these sensors are too expensive. Alternatively, tubing is used to bridge the distance between 
a pressure tap to a pressure sensor. The system's response highly depends on tubing geometry and material (Bergh and Tijdeman 1965). The system's eigenfrequencies are reduced, and the phase lag is extended with increasing tubing length. The speed of sound in water is $a_{\text {water }} \approx 1450 \mathrm{~m} \mathrm{~s}^{-1}$, but can be greatly reduced due to the geometry and elasticity of the connector tube. Steel tubes are the superior choice to keep the speed of sound high. An example is given by Wulff (2006) based on Eq. 8, where $E_{\text {water }}$ is the volume modulus of water, $E_{\text {tube }}$ the volume modulus of the tube material, $D$ the tube's inner diameter, and $s$ the tube's wall thickness. For a tube with inner diameter of $4 \mathrm{~mm}$ and wall thickness of $1 \mathrm{~mm}$, the speed of sound reduces to $a_{\text {steel }} \approx 1390 \mathrm{~m} \mathrm{~s}^{-1}$ for steel and $a_{\text {polyamide }} \approx 630 \mathrm{~m} \mathrm{~s}^{-1}$ for polyamide tubing. Nevertheless, the speed of sound in water is larger than in air. Additionally, the time scale of flow structures in water is increased, and hence, the associated natural frequencies are reduced. Both the augmented speed of sound and reduced flow frequencies enhance the accurate determination of unsteady pressures.

$a_{\text {sound }}=\sqrt{\frac{1}{\rho_{\text {water }}\left(\frac{1}{E_{\text {water }}}+\frac{1}{E_{\text {tube }}} \cdot \frac{D}{s}\right)}}$

Care must be taken when setting up the pressure measurement system. The tubing and sensor cavity need to be filled completely with water. Any air bubble entrapped in the pressure line can introduce significant errors due to inertia effects and sloshing water (Sect. 3.2.1). A procedure on how to properly de-air the tubing system is provided in Sect. 3.2.2. Additionally, the tubing filled with water needs to be fully enclosed and not exposed to the flow to avoid pressure variation caused by the CoRIOLIs effect. Differential pressure sensors are utilized for the current investigation, and their practicality is assessed qualitatively. These sensors are chosen because they had been used in previous steady velocity experiments. Furthermore, their affordability allows the implementation of a dense sensor distribution in one model, and they can be easily installed in every model equipped with pressure taps. A viable alternative to the differential pressure sensors used in this study is presented by Kirk and Jones (2019) who use a set of surface mounted sensors for measurements on a surging airfoil in a towing tank.

\subsubsection{Inertia effects}

In a towing tank, the unsteady freestream velocity is achieved by moving the model. Therefore, sensors installed in the model also experience this movement relative to the quiescent working fluid. A simple experiment is conducted to showcase the existence of inertial forces that potentially falsify the pressure readings. A pressure sensor is mounted on the measurement platform outside of the model. The pressure sensor is arranged in a similar fashion as it is installed in the model. The reference side is filled with air and connected to an enclosure with venting holes. Thus, atmospheric pressure is measured. A proper enclosure for the reference pressure is crucial to avoid any ambient effects since the entire setup is moved. The pressure side of the sensor is filled with water. A plug is inserted at the end of the pressure line to decouple the measurements from aerodynamic effects. Three configurations are tested, which are depicted in Fig. 5. In configuration (a), the pressure sensor is placed such that the pressure tubing is perpendicular to the velocity vector of the carriage train. The sensor is positioned so that the membrane experiences no deflection due to the carriage motion. An air bubble is purposely introduced into the pressure line. In configuration (b), the pressure sensor and tubing are rotated clockwise by $90^{\circ}$ such that the tubing is parallel to the carriage velocity vector. The pressure sensor membrane is allowed to have maximum deflection since the normal vector of the membrane's surface is aligned with the carriage velocity vector. An air bubble is purposely introduced in this configuration as well. Configuration (c) is similar to configuration (b) with the difference that no air bubble is introduced into the pressure line. Inertia effects are qualitatively determined when moving with the carriage train. The plot in Fig. 5 shows the pressure reading for all three configurations. The acceleration of the sinusoidal velocity profile is also included on a second y-axis. Configuration (a) (blue solid line) shows a negligible change in pressure during the measurements. The water in the tubing only sloshes in between the tubing walls due to the induced inertia forces. However, a sloshing motion up- and downwards is required

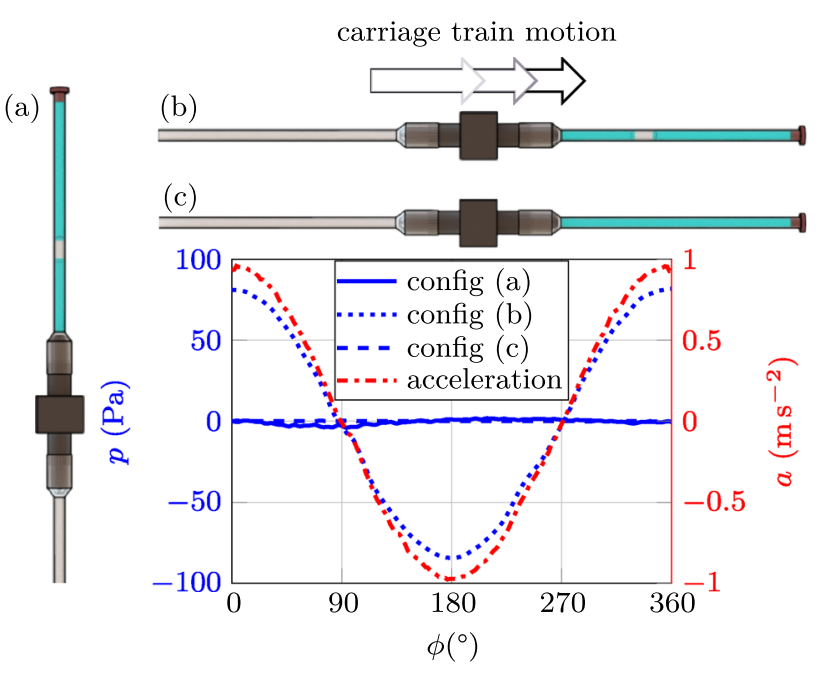

Fig. 5 Inertia effects depending on tubing orientation and entrapped air at $U_{0}=2.5 \mathrm{~m} \mathrm{~s}^{-1}\left(R e_{0} \approx 1,100,000\right), f=0.125 \mathrm{~Hz}(k=0.08)$, and $\sigma=0.5$ 
to deflect the sensor's membrane in this setup. Significant pressures changes are recorded for configuration (b) (blue dotted line). The water in the pressure tubing sloshes left and right due to the air pocket, thus deflecting the sensor's membrane. The pressure reading follows the acceleration profile. This validates the existence of inertia effects. No inertia effects are evident in configuration (c). The water cannot slosh sideways without any compressible air bubbles, and thus, no membrane deflection is imposed.

Results show that inertia effects are introduced when the pressure line is not completely filled with water, the model is moving, and the orientation of pressure line and sensor is not optimal. Further, the amplitude of inertial forces depends on the instantaneous acceleration. Therefore, pressure coefficients at low velocity but high frequency are burdened by larger relative errors than high velocity cases. Inertia effects in the pressure signal are avoided by appropriately positioning the pressure sensor and its tubing as in configuration (a). However, other effects leading to pressure distortion may still occur if air is entrapped in the tubing. The pressure signal will partially be transmitted and reflected on the air-water interface. The speed of propagation is reduced. Additionally, the entrapped air will expand and compress depending on the instantaneous pressure acting at the pressure tap location. Therefore, nonlinear effects may be present, especially with surging freestream velocity. The effects of bubble size, tube material, and tube cross section are not investigated in the presented work. An in-depth study including a dynamic pressure calibration in water needs to be carried out to quantify and prevent these effects.

\subsubsection{De-airing of pressure sensors}

De-airing of the pressure sensor cavity and tubing is crucial to obtain correct pressure readings as discussed in Sect. 3.2.1. Air bubbles in the pressure line or water drops in the reference line can introduce significant errors due to the impedance difference of the two media and their unique characteristics. A procedure is suggested to ensure that no air bubble is enclosed in the pressure line connected to the pressure tap that needs to be completely filled with water.

The reference side of the differential pressure sensors is connected to a tube filled with air. This tube is inserted into the reference enclosure located on the measurement platform outside of the water basin. It has to be ensured that no water penetrates the tube. Any water droplet will clog the reference pressure line. The opposite side of the pressure sensor is connected with water filled tubing. However, prior to that, it is important to slowly fill the pressure sensor cavity with water before submerging it. This is done by taking a syringe with a fine needle and carefully filling the cavity. A fine needle that dispenses very small water droplets is crucial.
Big droplets increase the chance of entrapping air during the filling process. Only thereafter the pressure sensor is submerged under water. Skipping this step highly increases the probability of entrapping air in the cavity due to surface tension effects and the small orifice. Shaking and tapping the sensor may help to remove enclosed air. However, it is not possible to guarantee complete air removal since the sensor and its fittings are not translucent. Additionally, shaking the sensor may damage the membrane. In the next step, the tubing is connected to the hypodermic needle of the pressure tap. A syringe is used to push water through the tube system until all air is removed. Then, the tubing is connected to the pressure sensor, while both are submerged and filled with water. This specific procedure allows for repeatable measurement of unsteady pressure in water with this type of sensor.

Measurements are repeatable over several weeks during a measurement campaign. However, based on discussions with other researchers the authors came to the awareness that this is not always the case. Often, air bubbles are found in the pressure line later on during the experiment. Water always contains dissolved gases, and degassing occurs when water temperature increases. Therefore, air bubbles can intrude into the tubing system over time due to temperature fluctuations. One tiny gas bubble potentially blocks the entire tubing, especially when using tubing with a small inner diameter. Kume et al. (2006) use degassed water to fill the vinyl tubes, which is likely done by pre-heating the water. Alternatively, silicon oil may be used to fill the sensor cavity because it is a good pressure transmitting fluid. Wulff (2006) utilizes silicon oil to prevent leaking of fluid in the recess in front of a surface mounted pressure sensor when changing configurations. In the present study, silicon oil (50 CST) is used to fill the pressure sensor cavity with a syringe as explained before. It is intended to remove air from the sensor cavity more reliably due to its low viscosity. Compared to water at $25^{\circ} \mathrm{C}$, the chosen oil (50 CST) has reduced surface tension but similar density ( $\rho_{50 \text { CST }} / \rho_{\text {water }} \approx 0.96$ ). No difference in pressure recording is observed when using silicon oil or water to fill the sensor cavity. In the current study, the reliability of this method was not assessed in detail especially when the tubing system is completely filled with silicon oil. It is advised to check the compatibility of the pressure sensor and the oil. For example, a different silicon oil (0.65 CST) was also used in this study because of its low surface tension but resulted in destruction of a pressure sensor. Water is used for future studies in this facility since temperature changes over the time span of a measurement campaign are negligible due to the bulk of water in the basin. 


\subsection{Unsteady model velocity and test rig vibration}

Surge experiments are often carried out in wind tunnels where louver vanes are used to create the unsteady freestream velocity. In many cases, it is not feasible to reach high ReYNOLDS numbers Re, reduced frequencies $k$, and velocity amplitudes $\sigma$ due to inherent challenges of the underlying facility. Using a towing tank instead of a wind tunnel increases the range of these parameters by simply controlling the electrical motors. The importance of obtaining an accurate velocity profile is discussed in Sect. 3.3.1. A simple but effective iterative scheme is provided to increase the accuracy of the carriage train velocity profile. Proper synchronization of signals is crucial for correct results.

Contrary to wind tunnel tests, the entire test rig moves through a water basin when using a towing tank. Section 3.3.2 offers some means for vibration detection and a procedure on how to distinguish mechanical from flowinduced frequencies.

\subsubsection{Accuracy of velocity profile and feasible range of parameters}

The velocity of the carriage train is controlled by feeding the carriage controller with the desired input signal that is derived from steady operations. Based on Eqs. 1-5 with a maximum carriage acceleration of $a=1 \mathrm{~m} \mathrm{~s}^{-2}$ for this facility, the theoretical parameter range for oscillatory motion profiles can be determined. The transfer function describing the relationship between voltage input and carriage velocity is calibrated with constant velocities and results in a linear relationship. The obtained calibration coefficients are then used to calculate the required voltage input to attain the desired velocity profile for the carriage as defined in Eq. 2. Initial tests reveal that this transfer function is not sufficient for unsteady velocity profiles. Nonlinearities occur, and deviations between desired and actual velocity profile are apparent (Fig. 7a). The distortions highly depend on the chosen control parameters of the towing carriage motion such as mean velocity $U_{0}$, frequency $f$, and relative velocity amplitude $\sigma$. The deviations between input and output signal increase especially for low carriage velocities when the DC-motors operate at off-design points as well as for high forcing frequencies $f$. Figure 6 shows the corresponding envelopes with lines of constant REYNOLDS number Re according to Eq. 5 and model chord length of $c=0.5 \mathrm{~m}$. Red and green dots highlight test cases where the actual carriage velocity profile exhibits an instantaneous relative error $\left(\epsilon_{\text {rel }}=\left[\left(U(t)_{\text {model }}-U(t)_{\text {desired }}\right) / U(t)_{\text {desired }}\right] \cdot 100\right)$ of more and less than $5 \%$ compared to the desired signal.

The accuracy of the towing carriage velocity is increased by implementing an iterative scheme that takes any nonlinearities into account and is readily

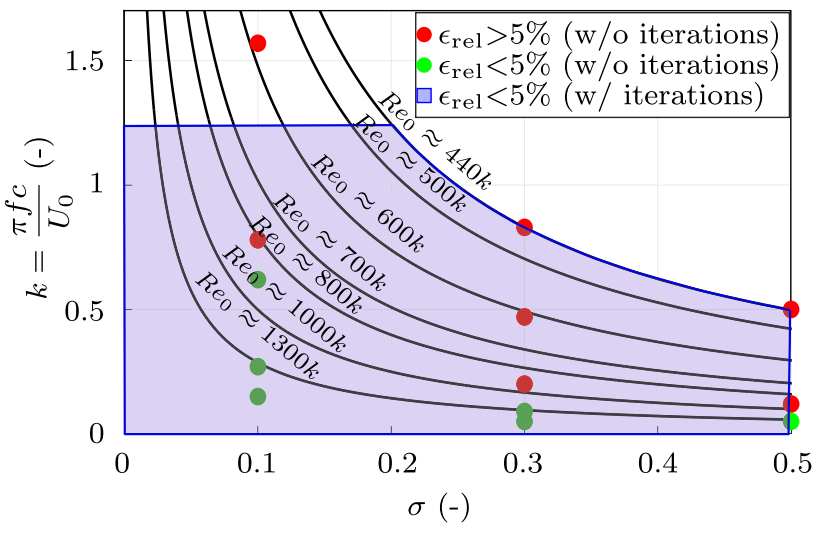

Fig. 6 Feasible range of parameters for lines of constant mean ReYNOLDS number $(c=0.5 \mathrm{~m})$

implemented. The feasible parameter space with an instantaneous relative error below 5\% is enlarged and denoted by the blue shaded area in Fig. 6. Based on the absolute error between input signal and actual carriage velocity, a new function is generated that compensates exactly for the error at each point measured. Figure 7 a shows the desired output signal with a red dotted line, which is identical to the controller input signal, describing a sinusoidal motion $U(t)=U_{0}(1+\sigma \sin (\omega t))$, where $U_{0}=1 \mathrm{~m} \mathrm{~s}^{-1}$ $\left(R e_{0} \approx 440,000\right), f=0.31 \mathrm{~Hz}(k \approx 0.49)$, and $\sigma=0.5$. The actual carriage velocity is shown by a black solid line. The relative error, indicated by the green dash dotted curve, reaches almost $20 \%$ in the second half of the motion cycle. Applying the iterative approach and carrying out six iterations, a new highly nonlinear input signal is created as is shown by the blue dashed line in Fig. 7b. The maximum relative error is substantially decreased to a value of $1.6 \%$. The number of iterations necessary to converge to a user-defined threshold is dependent on the mean velocity $U_{0}$, frequency $f$, and relative velocity amplitude $\sigma$ of the desired velocity profile. Only two iterations are necessary when $U_{0}=3 \mathrm{~m} \mathrm{~s}^{-1}, f=0.1 \mathrm{~Hz}$, and $\sigma=0.5$ are chosen to produce an actual carriage velocity profile with deviations from the desired signal of less than $2 \%$. The repeatability of the actual velocity profiles using a nonlinear distorted input signal is excellent, and no cycle-to-cycle variation is experienced. Tests are conducted with and without the model installed at various angles of attack. It is concluded that unsteady loading on the model has negligible effect on the accuracy of the carriage velocity. However, this may be different for other facilities, especially in smaller towing tanks.

Theoretically, any arbitrary function can be generated as an input signal. For sinusoidal velocity tests, it is best practice to apply an acceleration matching at the beginning 


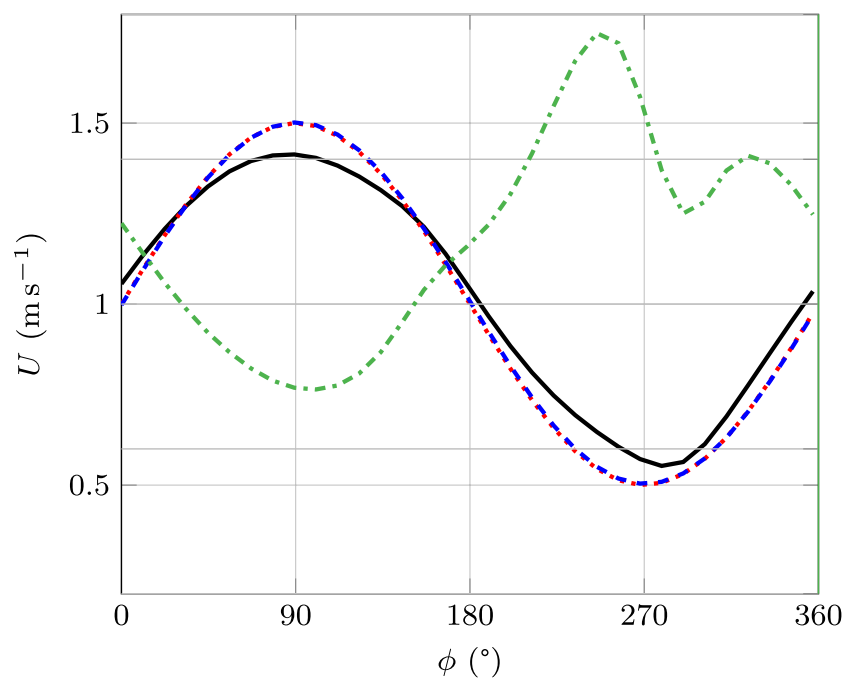

(a) No iteration. Input signal equals desired signal $U(\phi)$

Fig. 7 Iterative procedure to increase the accuracy of the model velocity

and end of the oscillatory phase to obtain a continuous and differentiable signal that guarantees a smooth transition between all three phases of a test run (i.e., acceleration, oscillation, and deceleration). Equation 3 can be used to calculate the required initial acceleration and final deceleration. The sinusoidal signal has to be extended by half a period at the end of a run to apply this matching principle.

Flow quantities are usually averaged over a long period of time when conducting experiments at constant velocity. This is not possible for unsteady measurements where quantities need to be known at distinct time instances. Therefore, it is crucial to verify that all captured signals are synchronized in time. Often, when quantities are recorded simultaneously, synchronization is taken for granted. However, this cannot always be guaranteed. For this particular setup, the frequency-to-current converter utilized introduced a time delay in the velocity signal that needs to be accounted for. Since pressure and velocity change nonlinearly in time, results are not only phase shifted but also changed in magnitude leading to severe alteration of the data's dynamic response. In future studies, a more sophisticated transistor-transistor logic (TTL) converter will be used that transforms the signal of the rotary encoder in less than $100 \mathrm{~ns}$.

\subsubsection{Setup-induced vibration}

Various sources of vibration can affect the experiments in a water towing tank. The test rig and model are installed on a measurement platform and moved together through the water basin. Any vibration is transmitted through the facility in all directions. Eventually, vibrations are picked up by the

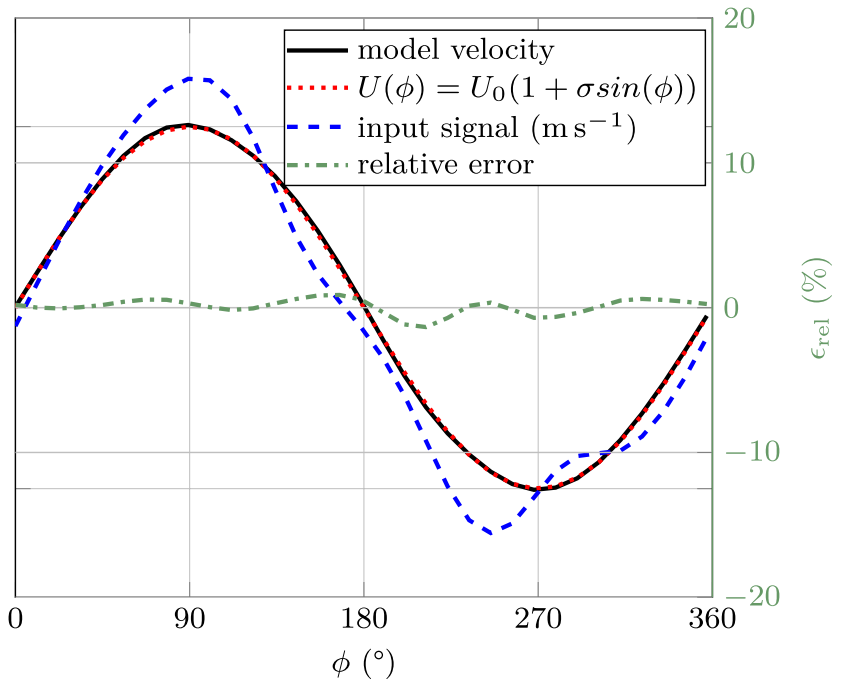

(b) Six iterations

model and sensed by the integrated sensors, thus influencing data quality. Furthermore, vibration can potentially influence boundary layer transition and separation. Therefore, it is important to detect and alleviate sources of vibration, and to distinguish between mechanical and flow-induced vibration. With this knowledge, the setup can be improved and the data can be filtered appropriately. Adequate filtering is important to identify outliers accurately.

Hence, a procedure is developed that allows for pure vibration identification independent of model and facility. Accelerometers (acc) are a viable tool to identify vibration and can be placed anywhere on the test rig or model. Various configurations are tested: nothing installed (baseline), test rig installed but no model, and finally the installation of both. Results are shown for unsteady model velocities only. All results discussed within this section are unfiltered phase averaged signals. Changes in the accelerometer signal are detected and compared between the tested configurations. Power spectral density (PSD) spectra of the phase averaged acceleration and pressure signal are presented in Fig. 8 . Vibrations are apparent for the baseline test case when no test rig is installed (red, dashed line) due to the operation of the DC-motors and contact of the carriage train wheels with the train tracks. Additional peaks occur when the test rig is installed (brown, solid line). Different spectral content is distinguished in the accelerometer (blue, dotted line) and pressure (black, dash dotted line) signal after installing the model at an angle of attack of $\alpha=0^{\circ}$. Yet, frequencies cannot be distinguished with certainty. The reference velocity of the model changes dynamically when performing unsteady measurements. Therefore, velocity-dependent frequencies change instantaneously leading to a distorted 


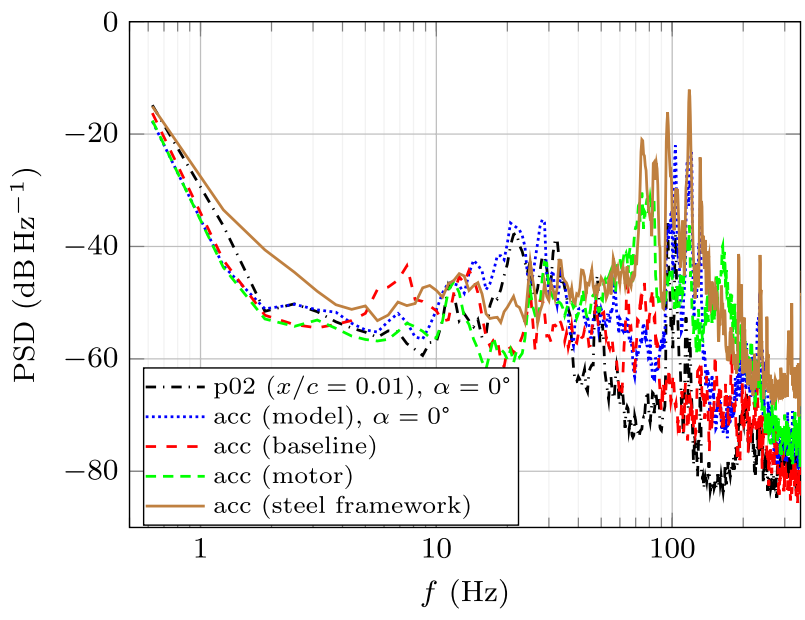

Fig. 8 Power spectral density spectrum of different signals at $U_{0}=2.5 \mathrm{~m} \mathrm{~s}^{-1}\left(R e_{0} \approx 1,100,000\right), f=0.125 \mathrm{~Hz} \quad(k=0.08)$, and $\sigma=0.5$

frequency spectrum where peaks get smeared out. Furthermore, vibrations that occur only over a short instance may not be captured due to little weight compared to the entire length of the signal.

A better way to analyze the frequency content in the acquired signals is to calculate the short-time Fourier transform (STFT) and plot the results in a spectrogram. This captures frequency and magnitude as a function of time such that time-dependent frequencies are detected. Figure 9 shows various spectrograms for different configurations. The baseline noise when no test rig and model are installed is shown in Fig. 9a. The signal is acquired using an accelerometer attached to the measurement platform closest to the point where the test rig would be mounted. Frequencies ranging from $40-80 \mathrm{~Hz}$ are detected that scale with the unsteady velocity profile. Comparing this signal to Fig. 9 , where an accelerometer is placed directly on one of the driving motors, the same frequency content is found, among others, but highly amplified in magnitude. The instantaneous frequencies scale with the driving frequency of the eight pole motor as the 8th superharmonic. Those frequencies are attributed to motor-induced vibration.

In the next step, the test rig is mounted. An accelerometer is attached to the front right steel framework, and the corresponding spectrogram is provided in Fig. 9c. Strong vibrations that scale with the carriage velocity occur in the first half of the motion cycle as long as a velocity of $2 \mathrm{~m} \mathrm{~s}^{-1}$ is exceeded. Since no model is installed, these vibrations are caused by the submerged test rig that is towed through the water basin. A constant Strouhal number of $S t \approx 0.2$ is calculated in the first temporal half of the spectrogram when normalizing the spectrum with the thickness of the welded steel plates and instantaneous velocity. Thus, the vibrations are caused by wake shedding vortices, which separate from a multitude of thin steel plates. While wake shedding cannot be prevented entirely, it can be reduced by properly profiling the submerged parts. Furthermore, knowing that vibration caused by the submerged parts can become excessively large, it is important to make appropriate design considerations. The thickness of these parts, if possible, should be chosen such that induced vibrations are shifted to higher frequencies compared to the model's characteristic frequencies. Model shedding frequencies as well as vibrations introduced by other sources are captured by placing an accelerometer into the model (Fig. 9c). Previously identified motor and setup-induced vibrations are transferred to the model. Additionally, frequencies below $f=40 \mathrm{~Hz}$ are detected that are not observed in any other spectra. Figure 9e shows the spectrogram of a pressure sensor installed in the leading edge of the model located at $x / c=0.01$. A frequency band ranging from $10-30 \mathrm{~Hz}$ is detected, which scales with the instantaneous carriage velocity. When scaled with the model's thickness, an instantaneous Strouhal number of approximately $S t \approx 0.2$ is calculated, which remains constant over the entire period. This indicates the occurrence of model shedding frequencies associated with wake vortices, which scale with the freestream velocity. However, the most energetic aerodynamic frequency is found at the forcing frequency of the velocity profile. This frequency cannot be properly resolved by analyzing the phase averaged signal. Therefore, lower frequencies appear as a dark line in the spectrogram. Apart from that, the setup-induced frequencies in the range of $100-140 \mathrm{~Hz}$ are the most dominant frequencies that are translated to the model, thereby affecting the data quality of the pressure measurements.

These setup-induced vibrations are of the same order of magnitude as the wake vortices shed from the model. In this example, it is the wake vortex shedding of the test rig, which has the strongest parasitic influence. However, this does not have to be true for all parameter configurations. For velocity profiles with lower mean REYNOLDS number, motor vibrations are identified as the most influential source of vibration. Therefore, accelerometers should always be attached to the setup and model during testing in order to capture vibration sources that are not caused by the flow field around the model.

After being able to distinguish setup-induced vibration from model flow field frequencies, the setup should be improved by either profiling submerged parts of the test rig, changing the thickness of most influential parts to shift test rig shedding frequencies, or introducing appropriate vibration dampening material. Only thereafter, if no further improvements are achieved, appropriate data filtering can be applied without over/under filtering the signal. 


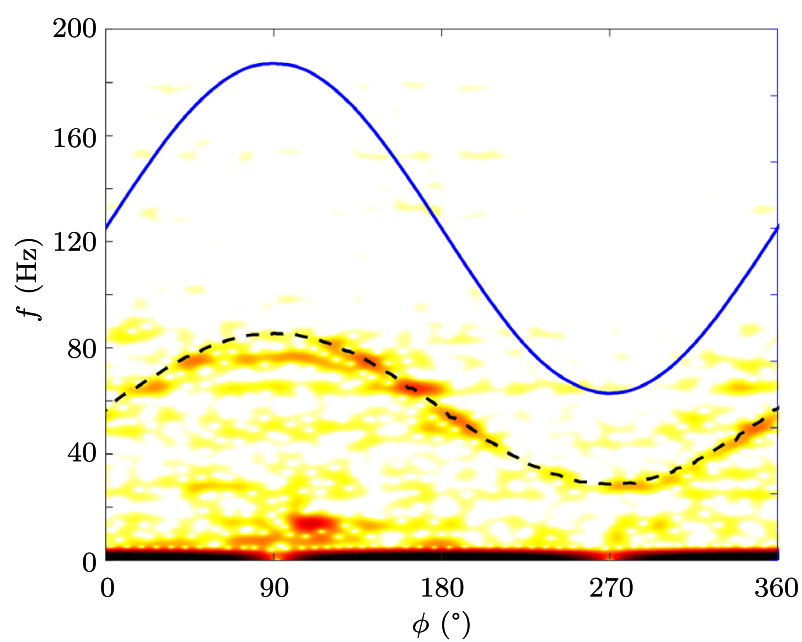

(a) No test rig installed (acc)

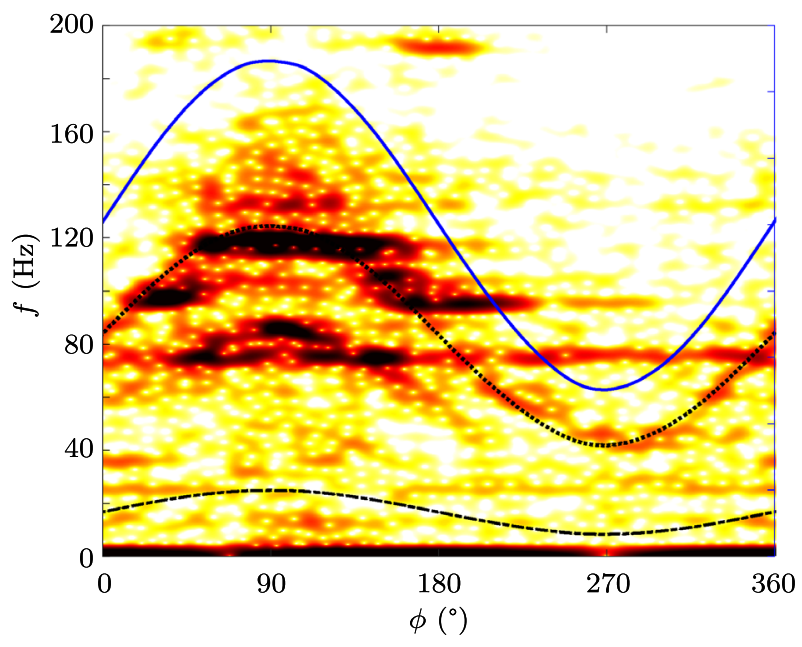

(c) Test rig installed but no model (acc)

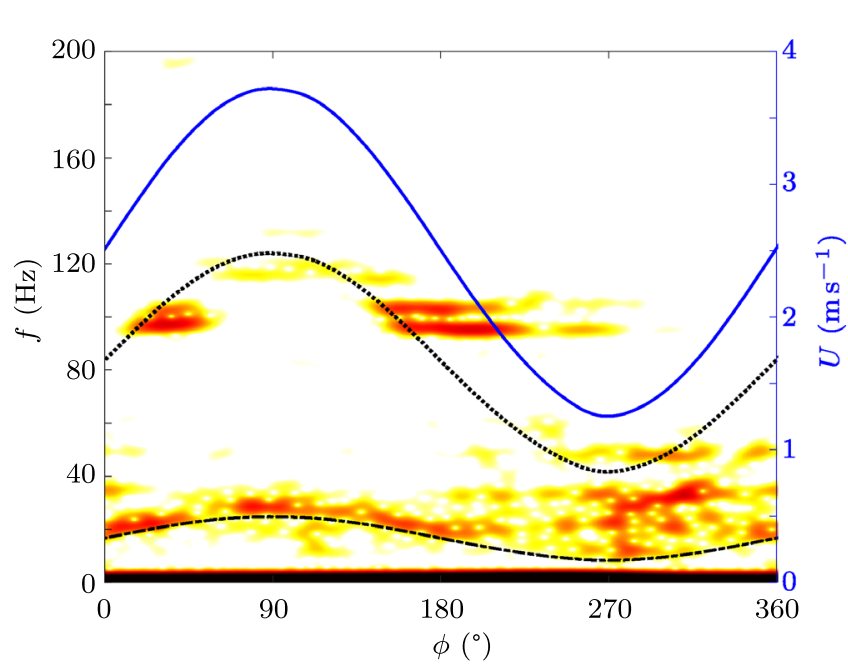

(e) Model (pressure sensor)

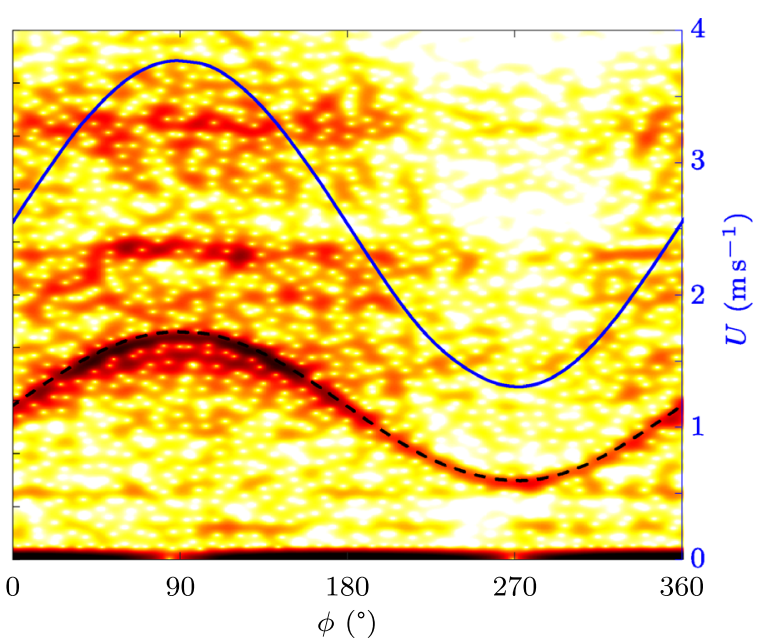

(b) Motor (acc)

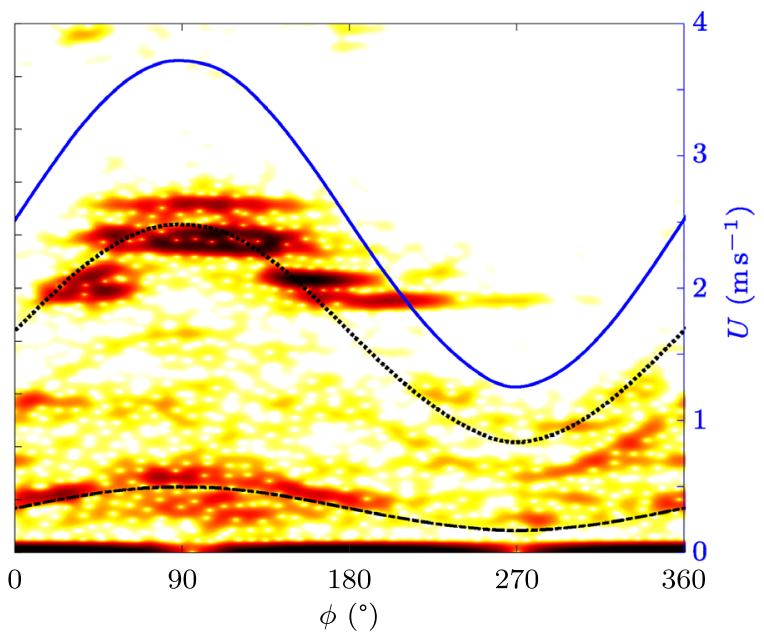

(d) Model (acc)

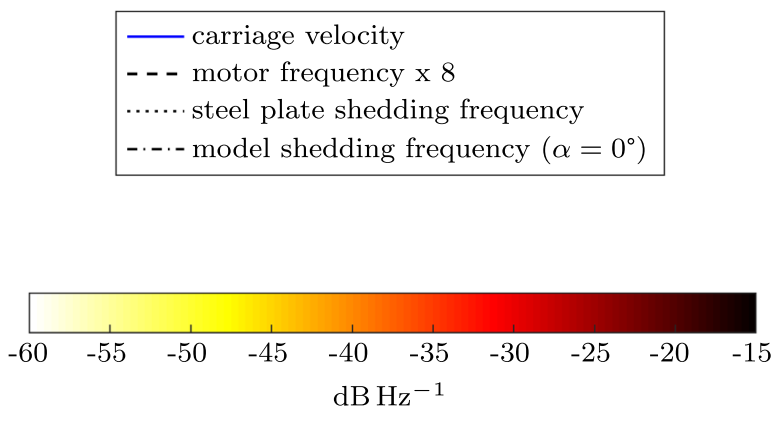

Fig. 9 Spectrograms of the accelerometer and pressure sensor signal for different setup stages at $U_{0}=2.5 \mathrm{~m} \mathrm{~s}^{-1}\left(R_{0} \approx 1,100,000\right)$, $f=0.125 \mathrm{~Hz}(k=0.08)$, and $\sigma=0.5$ 
Additional sources of vibration may be present, which are imposed by the environment. These sources inherently depend on the facility and where it is located. Pressure measurements with sensors installed within the model are conducted over a long period of time $(24 \mathrm{~h})$, while the carriage train is at standstill. Three additional sources of vibration are identified: first the city train passing over a bridge that crosses the towing tank facility about midway; second people walking over the carriage train; third the compressor for the pneumatic brakes that turned on when a certain pressure threshold is surpassed. The take away from this exercise is not to walk around the carriage train during measurements and to keep the brake's compressor in check before conducting each test run. No influence on the data is detected by the trains passing by. However, it is a good practice to ascertain that these environmental vibration sources do not influence the data quality. A thorough investigation regarding vibration and noise is recommended for every facility.

\subsection{Surface waves}

Surface waves are always present when operating a towing tank. These waves are generated by the model as well as surface piercing objects such as the model mount. Therefore, a superposition of multiple waves is observed. The wave system is inherently dependent on the test rig and model geometry as well as the towing speed. Sloshing waves are excited based on the towing tank geometry, which affect sensor offset time (Sect. 3.4.1).

Free surface effects have been investigated in various studies on submerged bodies with different model geometries and steady freestream velocity. In particular, the wave resistance is affected depending on wave pattern and submersion depth. For a rectangular bluff body, results are unaffected by free surface effects as long as the model submergence satisfies $d_{\text {model }} / H_{\text {model }}>2.0$ and the depth-based FROUDE number $F_{d}=u / \sqrt{g d_{\text {model }}}<0.37$ (Aoki et al. 1992). These results are confirmed by Malavasi and Guadagnini (2007) and Malavasi and Blois (2012) who studied free surface effects combined with ground effects. Studies carried out on an autonomous underwater vehicle revealed a sensitive response of the drag component on the freestream velocity (Mansoorzadeh and Javanmard 2014). Plunging airfoils near the free surface were investigated numerically (Zhu et al. 2006) and experimentally (Cleaver et al. 2013). Negligible free surface effects are experienced for a submersion depth twice the chord length. However, the drag force is affected considerably when plunging is carried out in proximity to the water surface around a constant unsteady parameter of $\tau=U_{\infty} 2 \pi f / g \approx 0.25$. Measurements on a high-speed train in a shallow water towing tank were carried out by Tschepe et al. (2019). A critical length-based FroudE number range $0.2<F_{L}<1.2$ is identified in which the drag forces are affected substantially. A correction method is provided to allow measurements at wider FROUDE number range.

The transferability of these results obtained from steady velocity tests to the unsteady model motion remains questionable due to nonlinear interaction of surface waves and model velocity. Nevertheless, the main findings of the publications on constant velocity experiments are consistent with the water wave theory and suggest that free surface effects reduce with increasing model depth. However, practical constraints often limit submersion depths so that measurements are not completely independent of free surface or ground effects. In some instances, the addition of a skim plate is a practical solution for the alleviation of surface wave effects (Stephens et al. 2016; Stevens and Babinsky 2016; Stevens et al. 2017). To the best of the author's knowledge, there are no guidelines on how to design and utilize a skim plate appropriately. Furthermore, it appears that no study has been published yet that validates the effectiveness of a skim plate. Section 3.4.2 discusses preliminary observations of employing a skim plate.

\subsubsection{Settling and sensor offset time due to sloshing waves}

Experimental data quality in towing tanks benefits from reaching zero turbulence intensity between runs. However, an extended waiting time is required for the water to settle and reach zero turbulence levels. Figure 10 visualizes the results of a long-time measurement using a wave sensor. A settling time of more than six hours is required to reach quiescent conditions in the basin. This does not imply that turbulence will completely decay as well once surface waves have settled. For most experiments, such long waiting times are impracticable and more efficient means are required in order to execute multiple test runs within a day.

A great number of experiments are carried out with steady and unsteady model velocities with different waiting times ranging from 0 to 72 hours. For the tested model, the turbulence intensity and prevailing waves do not affect the (phase)-averaged quantities. Results are repeatable and

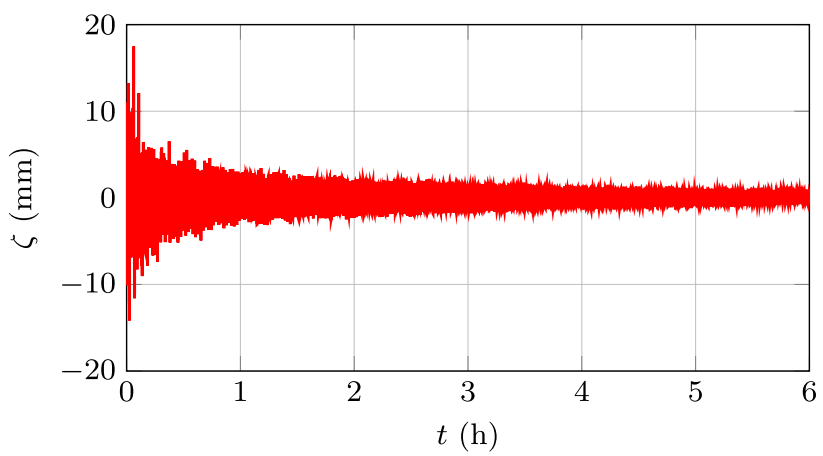

Fig. 10 Decay of sloshing wave amplitude $\zeta$ 


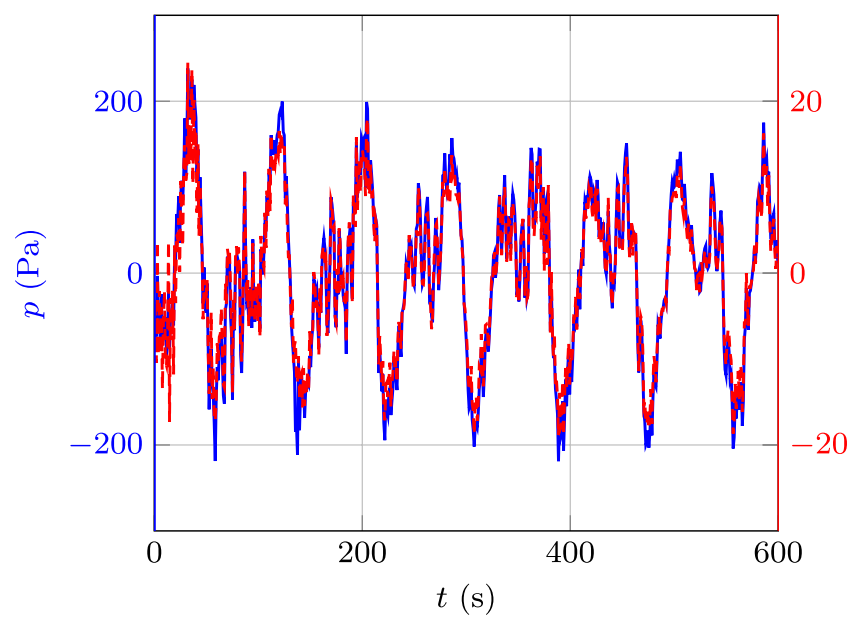

(a) Time series

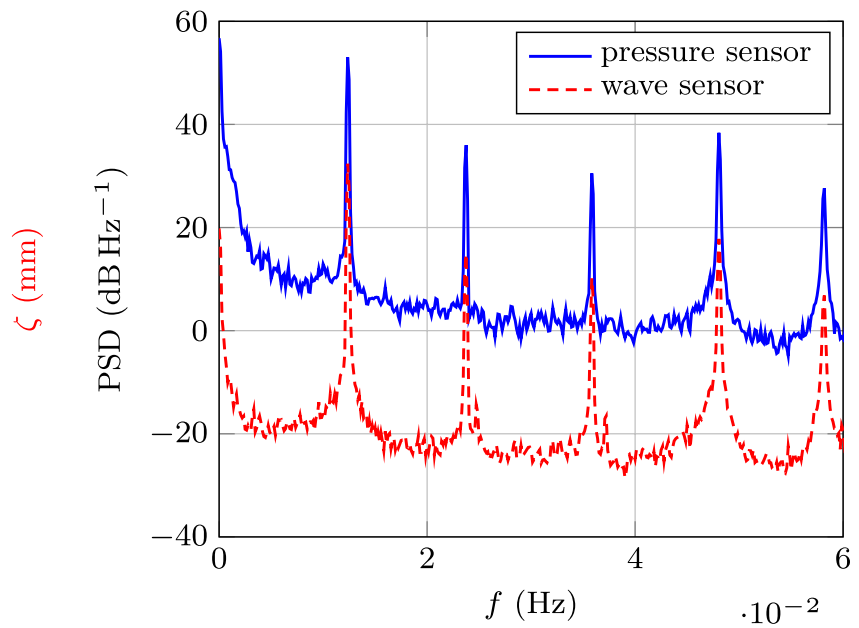

(b) PSD Spectrum

Fig. 11 Sloshing waves measured with pressure and wave sensor at $f=512 \mathrm{~Hz}$

Table 1 Frequency of sloshing wave modes determined theoretically $\left(f_{n}\right)$ and experimentally $\left(f_{\text {exp }}\right)$

\begin{tabular}{llll}
\hline $\mathrm{n}$-th mode & $\mathrm{f}_{\text {exp }}(\mathrm{Hz})$ & $\mathrm{f}_{\mathrm{n}}(\mathrm{Hz})$ & $\mathrm{f}_{\text {exp }} / \mathrm{f}_{\mathrm{n}}$ \\
\hline 1 & 0.0124 & 0.0124 & 1.0000 \\
2 & 0.0238 & 0.0248 & 0.9597 \\
3 & 0.0358 & 0.0371 & 0.9650 \\
4 & 0.0481 & 0.0493 & 0.9757 \\
5 & 0.0582 & 0.0614 & 0.9479 \\
\hline
\end{tabular}

independent of waiting time if data are recorded sufficiently long. Appropriate selection of sensor offset time is identified as the dominant factor to prevent scatter in the results.

Sloshing waves are excited by introducing perturbations into the water in the form of KeLvin wake waves. Figure 11a shows the recurrence of a wave pattern that is measured using a pressure sensor and a wave sensor, while the carriage train is not moving. Distinct modes are excited based on the towing tank geometry. For rectangular tanks, the n-th fundamental sloshing frequency is calculated by a closedform solution given in Eq. 9 (Jung et al. 2015), where $f_{n}$ is the $n$-th sloshing frequency $(\mathrm{Hz}), n$ the mode number, $g$ the acceleration due to gravity $\left(\mathrm{m} \mathrm{s}^{-2}\right)$, and $L$ and $h$ the water basin length and depth $(\mathrm{m})$. The analogous problem is the treatment of room acoustic modes in air. While surface deflection $\zeta$ is a function of towing speed and geometry of submerged parts, the wave modes are solely dependent on the water tank geometry. Table 1 shows the first five theoretical modes calculated using Eq. 9. The calculations are based on the local depth of $h=3.4 \mathrm{~m}$, where the wave and pressure sensor are positioned. The basin length of $L=233 \mathrm{~m}$ is obtained by excluding the length of the preparation area since it introduces significant changes in cross section area. A PSD spectrum of pressure and wave signal is presented in Fig. $11 \mathrm{~b}$ and shows the first five modes determined experimentally. The match of sloshing wave frequency obtained theoretically and experimentally is good considering that the shape of the water basin is not rectangular and the depth not constant along the basin's length. Deviations are below $6 \%$ (Table 1) and increase for higher wave numbers. The results indicate that shallow water approximation is applicable for sloshing waves everywhere in the water tank since the wavelength of the first modes is much greater than the basin or model depth. The water tank has solid wall boundaries at each end, and hence, the wavelength of the first mode must be $\lambda=2 L=466 \mathrm{~m}$. This result is verified using the shallow water approximation according to Eq. 10 where $T_{n=1}=1 / f_{n=1}$. Thus, hydrostatic pressure changes imposed by sloshing waves are measured everywhere equally and the prevailing waves can be measured using pressure sensors (e.g., sensor installed in the model).

$f_{n}=\frac{1}{2 \pi} \sqrt{\left.\frac{n \pi g}{L} \tanh \left(\frac{n \pi h}{L}\right)\right)}$

$\lambda_{1}=T_{1} \sqrt{g h}=466 \mathrm{~m}$

Exact knowledge about sloshing waves is important when these waves have not fully decayed. Routinely, a sensor offset is taken in order to compensate for any fluctuations in ambient condition (i.e., ambient pressure) and potential signal drift. The sensor offset is usually measured over a few seconds without much consideration of the exact duration. When taking the sloshing waves into consideration, the offset value may differ each time depending on the 


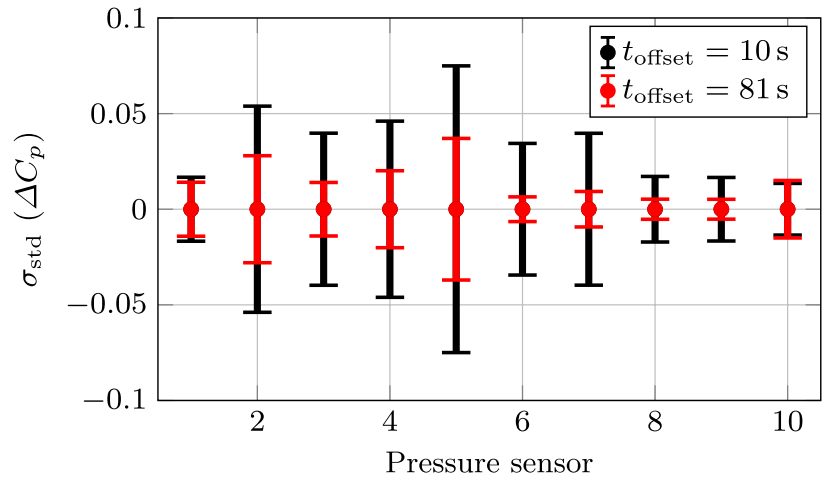

Fig. 12 Standard deviation depending on offset time at $\alpha=10^{\circ}$ and steady velocity $U_{0}=3 \mathrm{~m} \mathrm{~s}^{-1}\left(R e_{0} \approx 1,400,000\right)$ (waiting time in between test cases is $10 \mathrm{~min}$, each measurement repeated ten times, each measurement averaged over $30 \mathrm{~s}$ )

offset measurement length and its starting point relative to the sloshing wave period. Maximum discrepancies between offset values can range between twice the hydrostatic delta imposed by the sloshing wave. Therefore, the optimal choice for the offset time corresponds to the n-th multiple of the time period related to the first sloshing mode, which in this case is $T=1 / f_{n=1} \approx 81 \mathrm{~s}$. These observations are verified by measurements carried out at $\alpha=10^{\circ}$ and steady model velocities. Figure 12 shows the standard deviation of the pressure coefficient $C_{p}$ for ten pressure sensors. The settling time in between each measurement is set to ten minutes. A decrease in standard deviation is measured when increasing the offset time from $T_{\text {offset }}=10 \mathrm{~s}$ to $T_{\text {offset }}=T_{n=1}=81 \mathrm{~s}$. Solely the sensor closest to the trailing edge (pressure sensor 10) does not show any improvements because it is likely dominated by the unsteadiness of the wake shedding. An increase of the settling time to twelve hours or more did not alter the (phase)-averaged results for steady and unsteady measurements. Hence, choosing the sensor offset time appropriately can significantly increase the number of measurements per day while maintaining high accuracy of the obtained data.

Finally, turbulence and prevailing waves will always be present if settling times are not sufficiently long. Other than choosing the offset time appropriately, a good practice is to be consistent in all procedures. However, consistency does not mean to wait for a fixed period of time. The surface deflection and generation of turbulence depend on many test parameters. Also, additional turbulence may accumulate with each test run. Particle motion potentially induces changes in effective velocity and angle of incidence, which may be different every time an experiment is carried out even though settling time is kept constant. A threshold value of pressure or wave signal may be the more reliable method to define the starting point of the next test

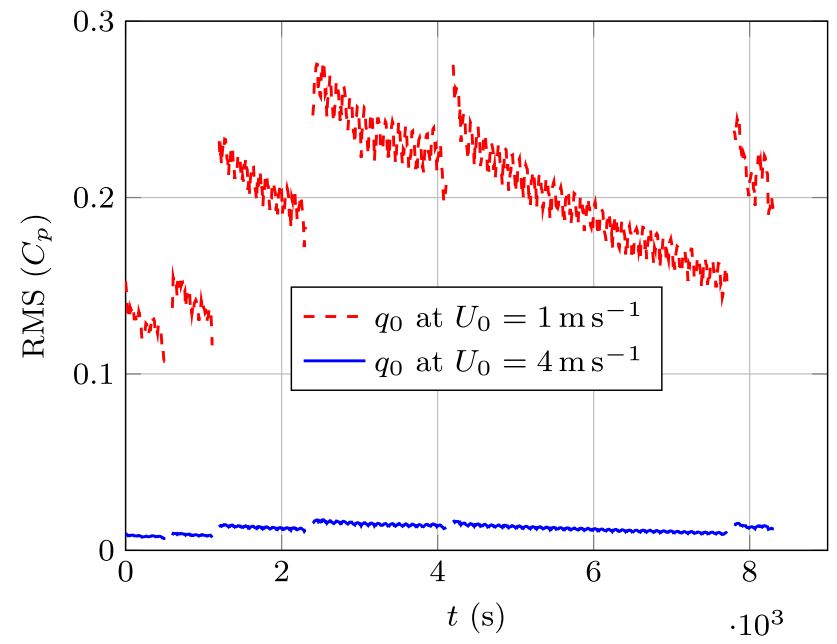

Fig. 13 RMS value of the pressure signal measured in the water basin in between test cases normalized by two different dynamic pressures $q_{0}$

run. Figure 13 illustrates the root mean square (RMS) values of a pressure signal monitored at an arbitrary position within the water basin in between several test cases. The window size for the moving average to calculate the RMS value is $T_{\mathrm{RMS}}=T_{\text {offset }}$. A decreasing RMS value over time is observed for all measurements. The magnitude of the RMS value depends on the previous tests conducted and the waiting times in between. The RMS value is always increased after a test run compared to the value before. The velocity when driving back into starting position is the same for all cases. However, results may be dependent on the relative position of sloshing waves and model motion when the model starts moving forward or backward in the water basin, which could explain the small increase in the overall RMS value after the second test is carried out (Fig. 13). Wave crests and troughs could cancel out when the backward motion is started appropriately offering an attractive possibility to reduce waiting times efficiently. While this is not tested, it is recommended to move back at low speed to avoid the generation of excessive waves with large amplitude. Furthermore, the waiting time will be a function of the subsequent test case. The (average) dynamic pressure $q_{0}$ offers a suitable choice to normalize the RMS value. Wave breakers are installed to accelerate the dissipation of waves, thus reducing the time a predefined RMS threshold value is reached. Even though settling time does not affect (phase)-averaged quantities in this study, it is expected to be dependent on model planform and shall be investigated on a case-by-case basis. 


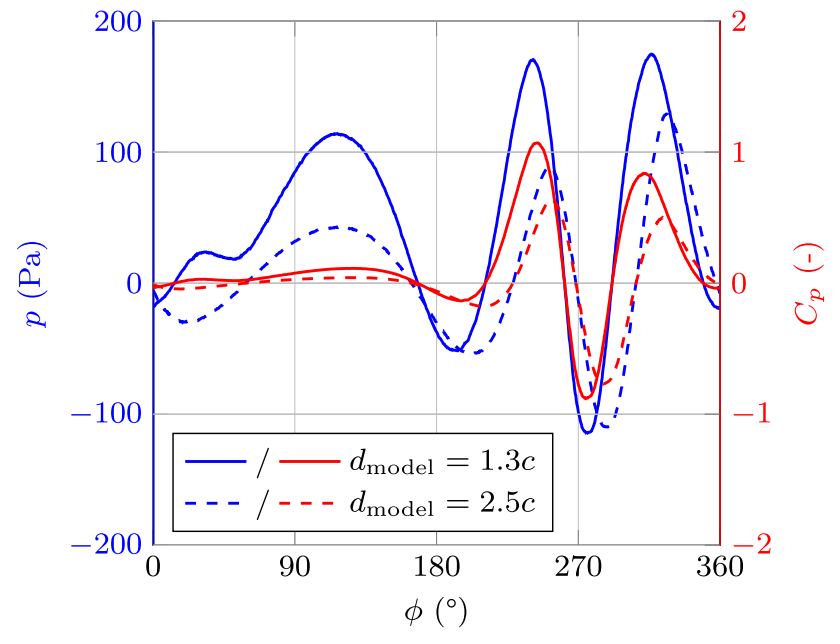

Fig. 14 Hydrostatic pressure variation measured with ultrasound wave sensor at $\alpha=10^{\circ}, U_{0}=1.0 \mathrm{~m} \mathrm{~s}^{-1}\left(R e_{0} \approx 440,000\right)$, $f=0.155 \mathrm{~Hz}(k \approx 0.24)$, and $\sigma=0.5$ for different model depths

\subsubsection{Model depth and skim plate}

Free surface waves and disturbances can affect the flow field around the submerged model. The induced particle motion and changes in hydrostatic pressure are reduced with increasing depth. Measurements with an ultrasound sensor are conducted to record the surface elevation during test runs. The sensor is mounted mid-chord above the model, fixed in its relative position to the model. Figure 14 shows the phase averaged results of the wave sensor for two different submersion depths. Blue lines depict changes in hydrostatic pressure based on the surface elevation. Red lines show the instantaneous pressure coefficient when the hydrostatic pressure is normalized by the instantaneous dynamic pressure. In particular, in the second half of the motion cycle, when the velocity is low, the instantaneous pressure coefficient reaches values of almost $C_{p}= \pm 1$. However, these changes are not necessarily imposed directly onto the model due to its submergence. Whether the perturbations introduced by the free surface affect the flow field around the model depends on the wave pattern generated during the test run (i.e., wave length of dynamic surface wave $\lambda$, wave amplitude $\zeta$, and submersion depth of the model $d_{\text {model }}$ ).

The unsteady tests demonstrate that the surface waves change dynamically (Fig. 14), and hence represent a timevarying boundary condition. The shape of these surface waves changes with the model's submersion depth. Therefore, the boundary condition is a function of the submersion depth when no skim plate is installed. It should be noted that different velocity profiles result in different wave patterns. In particular, when surging at high velocity amplitude $\sigma$, waves are produced that pass over the model entirely in the longitudinal direction.

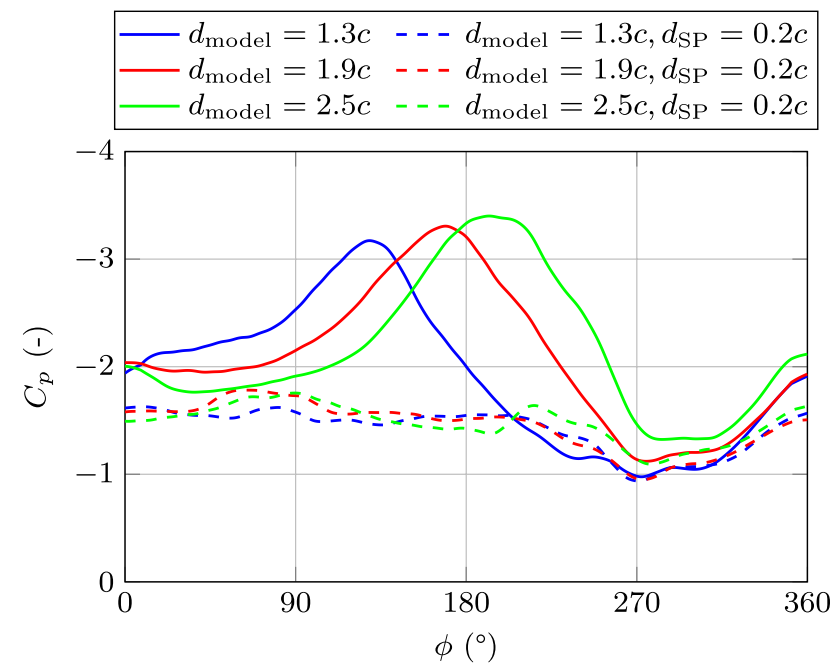

Fig. 15 Effect of model submergence on pressure signal at $x / c=0.04 \quad$ at $\quad \alpha=10^{\circ}, \quad U_{0}=1.0 \mathrm{~m} \mathrm{~s}^{-1} \quad\left(R e_{0} \approx 440,000\right)$, $f=0.155 \mathrm{~Hz}(k \approx 0.24)$, and $\sigma=0.5$

The submersion depth of the model is varied while keeping all other parameters constant to check for the influence of free surface effects. Results are expected to converge for increasing submersion depth to prove independence of surface effects. Figure 15 shows the phase averaged pressure signal of a pressure sensor positioned at $x / c=0.04$ when varying the model depth. Results are normalized by the instantaneous velocity. Solid lines represent the results without installation of a skim plate. All three curves show a distinct pressure minimum that occurs at higher phase angle for deeper model submergence. Deviations between results decrease with increasing model depth.

Dashed lines in Fig. 15 illustrate the influence of the skim plate, which is installed $0.1 \mathrm{~m}\left(d_{\mathrm{SP}}=0.2 c\right)$ below the water surface and centered above the model. The largest deflection of the surface waves for the investigated parameter configuration is measured to be $\pm 0.02 \mathrm{~m}$. The phase averaged surface pressure coefficients with skim plate installed demonstrate the effectiveness of this device. The variation of the pressure level during an entire period decreases significantly, and the pressure curves show good agreement independent of the model's submersion depth. A suction peak as noted without skim plate is absent. In the range of $210^{\circ}<\phi<330^{\circ}$ data with and without the skim plate installed show similarities and coincide.

It is concluded that a skim plate is necessary for repeatable and consistent data based on these results. However, a more in-depth study has to be carried out to remove existing ambiguities. For instance, the skim plate appears to be unnecessary for an angle of attack of $\alpha=20^{\circ}$, which is far beyond static stall, for steady and unsteady tests. No difference is measured with and without the skim plate for 
post-stall condition independent of the distance between skim plate and model in the range of $d_{\mathrm{SP}}=0.2 c-1.4 c$. However, it is possible that the skim plate induces a variation in angle of incidence, thus leading to the results as presented in Fig. 15. For $\alpha=10^{\circ}$ the skim plate could possibly increase the effective angle of attack to a regime (i.e., flow separation at the leading edge) where the skim plate remains ineffective. The induced effect on the angle of attack is confirmed by means of steady tests. For angles of attack ranging from $\alpha=5^{\circ}$ to $\alpha=10^{\circ}$, the pressure peak at the leading edge is enlarged when the skim plate is installed (not shown). However, without detailed study, it is assumed that a skim plate is an effective tool to diminish surface wave effects and should be used for all test cases. Regardless of the setup, it is advised to carry out measurements with model upside-up and upside-down to examine symmetry. For the presented investigation, the model was tested facing upside-down. An ongoing investigation focuses on the proper use of a skim plate for submerged airfoil models where symmetry is studied.

\section{Conclusion}

The presented work assesses the practicality and accompanying challenges of using a towing tank for steady and unsteady model motion for a submerged airfoil. The motivation for this study originated from the limited data on oscillatory surging flows, particularly at high REYNOLDS number Re, reduced frequency $k$, and velocity amplitude $\sigma$. Although towing tanks are being utilized for all kinds of experiments, various challenges exist that are either poorly documented or not addressed at all in the literature. Limited procedures and recommendations are available for the use of a water towing tank specifically when changing the freestream velocity as a function of time (i.e., surge). Despite the advantages compared to wind tunnels, the disadvantages of towing tank facilities must also be taken into account. The main focus of the presented work is to identify challenges for steady and unsteady model motion, to provide a set of initial guidelines and suggestions to enhance data quality, and to ensure validity of test results.

The limited basin length and indispensable settling time reduce the number of obtainable data sets. Therefore, data quality competes with available test time. The data quality of unsteady measurements is evaluated by means of the phase averaged pressure signal. Outliers are detected, and a convergence study is carried out. Initial transient effects are apparent within the first period of oscillation. The total number of required periods depends on the specific experiment to ensure data quality and test efficacy.

Pressure measurements are conducted with non-surface mounted pressure sensors as a lower cost alternative to surface mounted sensors. Signal distortion occurs when the attached tubing and sensor cavity are not fully de-aired. Inertia effects are observed when an air bubble is entrapped in the tubing system. The magnitude of inertial forces depends on the tube's orientation. A syringe is used to properly de-air the sensor cavity and its tubing. Additionally, it is recommended to use degassed water (preheated) or silicon oil (50 CST) to fill the tubing system if temperature changes are present.

The steady and unsteady motion is obtained by controlling the DC-motors of the carriage train. An iterative approach is presented that increases the accuracy of the performed motion. Nonlinear distortion of the velocity profile occurs, especially when the motors are operating at the lower end of the operating range. The error is minimized by iteratively adjusting the input signal according to the discrepancies between actual and desired velocity signal. The provided method can also be implemented for other model motions, such as pitch and plunge.

Several sources of mechanical vibrations are identified. Accelerometers attached to the model and support structures allow for vibration detection. Mechanical and flow-induced vibrations are distinguished by means of spectral analysis of the accelerometer and pressure signal. The unsteady signal is analyzed based on short-time Fourier transforms to preserve time information. Shedding frequencies are identified that scale with the instantaneous freestream velocity during unsteady surge motion. At high ReYNOLDS numbers, inevitable vortex shedding from the immersed test rig is the dominant source of vibration. Therefore, all components of the test rig exposed to the flow should be streamlined to reduce flow separation. The relevant length scales of the test rig and model should be different in order to avoid shedding at similar frequencies. The application of vibration dampening materials may help to further alleviate setup vibrations. Specific signal filtering is applied based on the spectral analysis. Independent of facility and steady or unsteady testing, it is highly recommended to use accelerometers to monitor the facility's vibrational response.

Surface waves at the air-water interface represent a major source for errors. Here, one can distinguish between two types of interference: first the development of so-called sloshing waves within the towing tank and second the formation of waves above the model, when performing a test run. Sloshing wave modes are excited by the introduced perturbations and are a function of the water basin's geometry. The wave height and mode frequency can be measured using pressure or other adequate wave sensors. The hydrostatic pressure along the basin is affected by the prevailing long scale waves. This affects settling time, and therefore the waiting time in between test cases. A predefined RMS value for wave settling has to be reached instead of waiting for a fixed period of time. The time it takes to record the 
offset of any sensor is also affected by sloshing waves. The offset should be taken according to the time period of the first sloshing mode if waves have not fully decayed. This ensures data accuracy and repeatability. Furthermore, surface waves change dynamically during the unsteady model motion which imposes a time varying boundary condition. Among other parameters, the wave pattern is a function of model submersion depth. A skim plate alleviates surface effects and is necessary for repeatable and consistent results.

Funding Open Access funding enabled and organized by Projekt DEAL.

Open Access This article is licensed under a Creative Commons Attribution 4.0 International License, which permits use, sharing, adaptation, distribution and reproduction in any medium or format, as long as you give appropriate credit to the original author(s) and the source, provide a link to the Creative Commons licence, and indicate if changes were made. The images or other third party material in this article are included in the article's Creative Commons licence, unless indicated otherwise in a credit line to the material. If material is not included in the article's Creative Commons licence and your intended use is not permitted by statutory regulation or exceeds the permitted use, you will need to obtain permission directly from the copyright holder. To view a copy of this licence, visit http://creativecommons.org/licenses/by/4.0/.

\section{References}

Aoki K, Miyata H, Kanai M, Hanaoka Y, Zhu M (1992) A waterbasin test technique for the aerodynamic design of road vehicles. In: International congress and exposition, SAE International

Bergh H, Tijdeman H (1965) Theoretical and experimental results for the dynamic response of pressure measuring systems. In: Nationaal Lucht-en Ruimtevaartcentrum (Nat. Aero- and Astronautical Res. Inst.)

Carr L, McAlister, KW, McCroskey W (1977) Analysis of the development of dynamic stall based on oscillating airfoil experiments. https://ntrs.nasa.gov/search.jsp?R=19770010056

Chen JH, Chang CC (2006) A moving piv system for ship model test in a towing tank. Ocean Eng 33:2025-2046. https://doi.org/10. 1016/j.oceaneng.2005.09.011

Cleaver D, Calderon D, Wang Z, Gursul I (2013) Periodically plunging foil near a free surface. Exp Fluids 54:21. https://doi.org/10. 1007/s00348-013-1491-9

Corkery S, Babinsky H, Harvey J (2018) On the development and early observations from a towing tank-based transverse winggust encounter test rig. Exp Fluids 59:398. https://doi.org/10. 1007/s00348-018-2586-0

Gad-el-Hak M (1987) The water towing tank as an experimental facility. Exp Fluids 5(5):289-297. https://doi.org/10.1007/ BF00277707

Greenblatt D, Müller-Vahl H, Strangfeld C, O1 M, Granlund K (2016) High advance-ratio airfoil streamwise oscillations: Wind tunnel vs. water tunnel. In: 54th AIAA Aerospace Sciences Meeting 2016, https://doi.org/10.2514/6.2016-1356

Gui L, Longo J, Stern F (2001) Towing tank piv measurement system, data and uncertainty assessment for DTMB Model 5512. Exp Fluids 31:336-346. https://doi.org/10.1007/s003480100293
International Towing Tank Conference (2020) Proceedings. https:// ittc.info/downloads/proceedings/

ITTC 75-01-03-03 (2008) Proceedings. https://ittc.info/media/1211/ 75-01-03-03.pdf

ITTC 75-01-03-04 (2014) Proceedings. https://www.ittc.info/media/ 7985/75-01-03-04.pdf

Jacobi G, Thill C, Huijsmans R (2016) The application of particle image velocimetry for the analysis of high-speed craft hydrodynamics. In: 12th International Conference on Hydrodynamics

Jung JH, Yoon HS, Lee CY (2015) Effect of natural frequency modes on sloshing phenomenon in a rectangular tank. International Journal of Naval Architecture and Ocean Engineering 7(3):580 594. https://ntrs.nasa.gov/search.jsp?R $=197700100560$

Kirk PB, Jones AR (2019) Vortex formation on surging aerofoils with application to reverse flow modelling. J Fluid Mech 859:59-88. https://ntrs.nasa.gov/search.jsp?R=197700100561

Kume K, Hasegawa J, Tsukada Y, Fujisawa J, Fukasawa R, Hinatsu M (2006) Measurements of hydrodynamic forces, surface pressure, and wake for obliquely towed tanker model and uncertainty analysis for cfd validation. J Mar Sci Technol 11(2):6575. https://ntrs.nasa.gov/search.jsp?R=197700100562

Leishman JG (2002) Challenges in modelling the unsteady aerodynamics of wind turbines. Wind Energy 5(2-3):85-132. https:// ntrs.nasa.gov/search.jsp?R=197700100563

Malavasi S, Blois G (2012) Wall effects on the flow structure around a rectangular cylinder. Meccanica 47(4):805-815. https://ntrs. nasa.gov/search.jsp? $\mathrm{R}=19770010056$

Malavasi S, Guadagnini A (2007) Interactions between a rectangular cylinder and a free-surface flow. J Fluids Struct 23(8):11371148. https://ntrs.nasa.gov/search.jsp?R=19770010056

Mancini P, Manar F, Granlund K, Ol MV, Jones AR (2015) Unsteady aerodynamic characteristics of a translating rigid wing at low REYNOLDS number. Phys Fluids 27(12):123102. https://ntrs.nasa. gov/search.jsp?R=19770010056

Mansoorzadeh S, Javanmard E (2014) An investigation of free surface effects on drag and lift coefficients of an autonomous underwater vehicle (AUV) using computational and experimental fluid dynamics methods. J Fluids Struct 51:161-171. https:// ntrs.nasa.gov/search.jsp?R=197700100567

McAlister KW, Carr LW, McCroskey WJ (1978) Dynamic stall experiments on the naca 0012 airfoil. https://ntrs.nasa.gov/ search.jsp?R=19770010056

Ostermann F, Woszidlo R, Nayeri CN, Paschereit CO (2015) Phaseaveraging methods for the natural flowfield of a fluidic oscillator. AIAA Journal 53(8):1-17. https://ntrs.nasa.gov/search. jsp?R=19770010056

Schmidt HJ, Woszidlo R, Nayeri CN, Paschereit CO (2017) Separation control with fluidic oscillators in water. Experiments in Fluids 58(8), https://doi.org/10.1007/s00348-017-2392-0

Stephens RG, Stevens PRRJ, Babinsky H (2016). A method for truck underbody aerodynamic investigation. https://doi.org/10.1016/j. oceaneng.2005.09.011

Stevens PRRJ, Babinsky H (2016) Experiments to investigate lift production mechanisms on pitching flat plates. Exp Fluids 58(1):7. https://doi.org/10.1016/j.oceaneng.2005.09.011

Stevens PRRJ, Babinsky H, Manar F, Jones A, Nakata T, Phillips N, Bomphrey R, Gozukara A, Granlund K, Ol MV (2017) Experiments and computations on the lift of accelerating flat plates at incidence. AIAA Journal https://doi.org/10.1016/j.oceaneng. 2005.09.011

Stevens RJ, Babinsky H, Manar F, Mancini P, Jones AR, Granlund KO, Ol MV, Nakata T, Phillips N, Bomphrey R, Gozukara A (2016) Low REYNOLDS number acceleration of flat plate wings at high incidence (invited). In: 54th AIAA Aerospace Sciences Meeting, American Institute of Aeronautics and Astronautics, Reston, Virginia, p 2016, https://doi.org/10.2514/6.2016-0286 
Tschepe J, Nayeri CN, Paschereit CO (2019) Analysis of moving model experiments in a towing tank for aerodynamic drag measurement of high-speed trains. Exp Fluids 60:110. https://doi.org/ 10.1016/j.oceaneng.2005.09.011

Tukker J, Blok JJ, Kuiper G, Huijsmans RHM (2007) Wake flow measurements in towing tanks with piv. In: 9th International Symposium on Flow Visualization

Wilhelmi H, Thieme T, Henning A, Wagner C (2018) Aerodynamic investigations of the effects of virtual coupling on two next generation trains. In: Dillmann A, Heller G, Krämer E, Wagner C, Bansmer S, Radespiel R, Semaan R (eds) 20. DGLR Fachsymposium der STAB, Springer, Notes on Numerical Fluid Mechanics and Multidisciplinary Design 136, vol 136, pp 695-704, https://doi.org/10.1007/978-3-319-64519-3_62
Wulff D (2006) Unsteady pressure and velocity measurements in pumps. In: Design and Analysis of High Speed Pumps (pp. 4-1 - 4-34). Educational Notes RTO-EN-AVT-143, Paper 4., https:// doi.org/10.1016/j.oceaneng.2005.09.011

Zhu Q, Liu Y, Yue DKP (2006) Dynamics of a three-dimensional oscillating foil near the free surface. AIAA Journal 44:2997-3009. https://doi.org/10.1016/j.oceaneng.2005.09.011

Publisher's Note Springer Nature remains neutral with regard to jurisdictional claims in published maps and institutional affiliations. 